\title{
Belnap-Dunn semantics for natural implicative expansions of Kleene's strong three-valued matrix with two designated values
}

\author{
Gemma Robles* and José M. Méndez** \\ *Dpto. de Psicología, Sociología y Filosofía, Universidad de León. \\ Campus de Vegazana, s/n, 24071, León, Spain. \\ gemma.robles@unileon.es; http://grobv.unileon.es. \\ ORCID: 0000-0001-6495-0388 \\ **Universidad de Salamanca. \\ Edificio FES, Campus Unamuno, 37007, Salamanca, Spain. \\ sefus@usal.es; http://sites.google.com/site/sefusmendez \\ ORCID: 0000-0002-9560-3327
}

\begin{abstract}
A conditional is natural if it fulfills the three following conditions. (1) It coincides with the classical conditional when restricted to the classical values $T$ and $F$; (2) it satisfies the Modus Ponens; and (3) it is assigned a designated value whenever the value assigned to its antecedent is less than or equal to the value assigned to its consequent. The aim of this paper is to provide a "bivalent" Belnap-Dunn semantics for all natural implicative expansions of Kleene's strong 3-valued matrix with two designated elements. (We understand the notion "natural conditional" according to N. Tomova, "A lattice of implicative extensions of regular Kleene's logics", Reports on Mathematical Logic, 47, 173-182, 2012.)

Keywords: Belnap-Dunn type bivalent semantics; Kleene's strong 3valued matrix; natural conditionals; 3 -valued logics; paraconsistent logics; logic of paradox.
\end{abstract}

\section{Introduction}

Let $T$ represent truth and $F$ represent falsity. Belnap-Dunn semantics (BDsemantics) is characterized by the possibility of assigning $T, F$, both $T$ and $F$ or neither $T$ nor $F$ to the formulas of a given logical language. BD-semantics originates with Belnap and Dunn's well-known logic B4 introduced to treat inconsistent and incomplete information (cf. [9], [10], [14] and [15]). The logic B4 is founded upon Smiley's 4-valued matrix MSm4, in its turn a simplification 
of Anderson and Belnap's matrix $\mathrm{M}_{0}{ }^{1}$. The logic $\mathrm{B} 4$ is equivalent to Anderson and Belnap's First Degree Entailment Logic, FDE (cf. [1], pp. 161-162).

Belnap and Dunn's approach has been generalized in the notion of a bilattice, which has found important applications in artificial intelligence (cf. [2], [3] and references therein).

Furthermore, Kleene's strong 3-valued matrix MK3 was defined in [20] in the context of the treatment of partial recursive functions. The matrix MK3 (our label) can be defined as shown in Definition 3.1 below. The connectives are conjunction, disjunction and negation. We can take either 2 as the only designated value or else both 1 and 2 . In the former case, 1 can be interpreted as neither truth nor falsity; in the latter, as both truth and falsity. The value 2 is, of course, truth, while 0 is falsity.

Finally, "natural conditionals", introduced in [34], are understood as stated in Definition 4.1 below. That is, a conditional is natural if the three following conditions are fulfilled. (1) It coincides with the classical conditional when restricted to the classical values $T$ and $F ;(2)$ it satisfies the Modus Ponens; and (3) it is assigned a designated value whenever the value assigned to its antecedent is less than or equal to the value assigned to its consequent ${ }^{2}$.

There are several possibilities for expanding the matrix MK3 with a conditional connective. For example, we can define the conditional with disjunction and negation similarly as in classical logic. Then, if 2 is the only designated value, the set of valid formulas is empty, but if 1 and 2 are designated, all (and only all) tautologies of classical logic are validated. Alternatively, the conditional can be introduced by means of an independent function. In this way, for example, Łukasiewicz's 3-valued matrix MŁ3 or the 3-valued matrix MRM3 can be defined ${ }^{3}$. MŁ3 (respectively, MRM3) is defined upon MK3 with only one (respectively, two) designated value.

In this paper, we shall consider all natural implicative expansions of MK3 with two designated values ${ }^{4}$. These expansions will be interpreted by means of a BD-semantics. There are two variants of BD-semantics, overdetermined BD-semantics (o-semantics) and underdetermined BD-semantics (u-semantics). Formulas can be assigned $T, F$ or both $T$ and $F$ in the former; $T, F$ or neither $T$ nor $F$ in the latter (cf. [29], [31]). The aim of this paper is to provide an overdetermined BD-semantics for all natural implicative expansions of Kleene's strong 3 -valued matrix with two designated values.

The present paper pursues previous work by the authors. In [31] and in [29], both an o-semantics and a u-semantics is provided for Lukasiewicz's 3-valued

\footnotetext{
${ }^{1}$ Actually, $\mathrm{M}_{0}$ is firstly defined in [8]; on MSm4 and its relation to B4, cf. [22] and references therein.

${ }^{2}$ Tomova adds a fourth condition: for any $a, b \in \mathcal{V}, a \rightarrow b \in D$, in other cases. We have dropped this condition in order to allow $a \rightarrow b \notin D$ even if $a, b \in D$ : the possibility of having non-designated conditionals when antecedent and consequent are dessignated is essential in some relevance and paraconsistent logics (cf. list of tables in section 4).

${ }^{3}$ MRM3 is the matrix determining the 3-valued extension of the quasi-relevant logic RM; cf. [1], [6], [11].

${ }^{4}$ In [30] all natural implicative expansions of MK3 with only one designated value are investigated.
} 
logic $\mathrm{E} 3$ and the 3 -valued logic $\mathrm{G} 3_{\mathrm{E}}$, respectively ${ }^{5}$; in [28] the logic G3 is endowed with a $\mathrm{u}$-semantics. These results connect 3 -valued logics with relevance logics; actually, with the most basic relevance logic, Anderson and Belnap's FDE and the BD-semantics devised for it.

The structure of this paper is as follows. In $\S 2$, we define some preliminary notions as used in the paper. Then, Belnap and Dunn's 4-valued matrix MB4 is defined. In $\S 3$, Kleene's strong 3-valued matrix is recalled and an overdetermined $\mathrm{BD}$-semantics is defined for K3, the logic characterized by MK3 when 1 and 2 are designated values. In $\S 4$, the notion of a "natural conditional" (according to [34]) is introduced and all natural implicative expansions of MK3 (with two designated values) are defined ${ }^{6}$. In $\S 5$, three basic logics are presented and some of their properties are proved. In $\S 6$, all the logics characterized by the natural implicative expansions of MK3 defined in $\S 4$ (let us name them Lt $i$ logics) are axiomatized as extensions of the basic logics. Most of these logics have not been investigated in the literature, as far as we know (cf. $\S 9$ ). In $\S 7$, an overdetermined BD-semantics is defined for each one of the Lt $i$ logics and the soundness theorems are proved. In $\S 8$, the completeness theorems are proved by means of a canonical model construction. Finally, in $\S 9$, the paper is ended with some concluding remarks on the results obtained.

\section{Belnap and Dunn's matrix}

In this section Belnap and Dunn's 4-valued matrix is recalled. Then, Kleene's strong 3-valued matrix MK3 along with a Belnap-Dunn semantics (BD-semantics) for the logic determined by this matrix can be defined in the following section.

We begin by stating some preliminary definitions where we recall some basic notions as used in the present paper. Next, Belnap and Dunn's 4-valued matrix MB4 is defined.

Definition 2.1 (Language) The propositional language consists of a denumerable set of propositional variables $p_{0}, p_{1}, \ldots, p_{n}, .$. , and the following connectives $\rightarrow$ (conditional), $\wedge$ (conjunction), $\vee$ (disjunction), $\neg$ (negation). The biconditional $(\leftrightarrow)$ and the set of wffs are defined in the customary way. $A, B$ etc. are metalinguistic variables.

Definition 2.2 (Logics) A logic $L$ is a structure $\left(\mathcal{L}, \vdash_{L}\right)$ where $\mathcal{L}$ is a propositional language and $\vdash_{L}$ is a (proof-theoretical) consequence relation defined on $\mathcal{L}$ by a set of axioms and a set of rules of derivation. The notions of 'proof' and 'theorem' are understood as it is customary in Hilbert-style axiomatic systems. $\Gamma \vdash_{L} A$ means that $A$ is derivable from the set of wffs $\Gamma$ in $L$; and $\vdash_{L} A$ means that $A$ is a theorem of $L$.

\footnotetext{
${ }^{5} \mathrm{G} 3_{\mathrm{E}}$ is a paraconsistent variant of 3 -valued Gödelian logic G3.

${ }^{6}$ We remark that there are stricter notions of "natural" in the literature; cf., e.g., [7].
} 
Definition 2.3 (Extensions and expansions of a propositional logic L) Let $\mathcal{L}$ and $\mathcal{L}^{\prime}$ be two propositional languages. $\mathcal{L}^{\prime}$ is a strengthening of $\mathcal{L}$ if the set of wffs of $\mathcal{L}$ is a proper subset of the set of wffs of $\mathcal{L}^{\prime}$. Next, let $L$ and $L^{\prime}$ be two logics built upon the propositional languages $\mathcal{L}$ and $\mathcal{L}^{\prime}$, respectively. Moreover, suppose that all axioms of $L$ are theorems of $L^{\prime}$ and all primitive rules of derivation of $L$ are derived rules of $L^{\prime}$. Then, $L^{\prime}$ is an extension of $L$ if $\mathcal{L}$ and $\mathcal{L}^{\prime}$ are the same propositional language; and $L^{\prime}$ is an expansion of $L$ if $\mathcal{L}^{\prime}$ is an strengthening of $\mathcal{L}$. An extension $L^{\prime}$ of $L$ is a proper extension if $L$ is not an extension of $L^{\prime}$.

Definition 2.4 (Logical matrix) A (logical) matrix is a structure $(\mathcal{V}, D, \mathrm{~F})$ where (1) $\mathcal{V}$ is a (ordered) set of (truth) values; (2) $D$ is a non-empty proper subset of $\mathcal{V}$ (the set of designated values); and (3) $\mathrm{F}$ is the set of $n$-ary functions on $\mathcal{V}$ such that for each n-ary connective $c$ (of the propositional language in question), there is a function $f_{c} \in \mathrm{F}$ such that $\mathcal{V}^{n} \rightarrow \mathcal{V}$.

Definition 2.5 (M-interpretation, M-consequence, M-validity) Let $M$ be a matrix for (a propositional language) $\mathcal{L}$. An M-interpretation $I$ is a function from $\mathcal{F}$ to $\mathcal{V}$ according to the functions in $\mathrm{F}$. Then, for any set of wffs $\Gamma$ and wff $A, \Gamma \vDash_{M} A$ ( $A$ is a consequence of $\Gamma$ according to $M$ ) iff $I(A) \in D$ whenever $I(\Gamma) \in D$ for all $M$-interpretations $I^{7}$.

Definition 2.6 (Belnap and Dunn's matrix MB4) The propositional language consists of the connectives $\wedge, \vee$ and $\neg$. Belnap and Dunn's matrix MB4 is the structure $(\mathcal{V}, D, F)$ where $(1) \mathcal{V}=\{0,1,2,3\}$ and it is partially ordered as shown in the following lattice

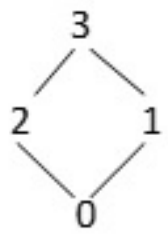

(2) $D=\{3,2\}$; (3) $\mathrm{F}=\left\{f_{\wedge}, f_{\vee}, f_{\neg}\right\}$ where $f_{\wedge}$ and $f_{\vee}$ are defined as the glb (or lattice meet) and the lub (or lattice join), respectively. Finally, $f_{\neg}$ is an involution with $f_{\neg}(0)=3, f_{\neg}(3)=0, f_{\neg}(1)=1$ and $f_{\neg}(2)=2$. For the reader's convenience, we display the tables for $\wedge, \vee$ and $\neg$ :

\begin{tabular}{|c|c|c|c|c|c|c|c|c|c|c|c|}
\hline$\wedge$ & 0 & 1 & 2 & 3 & $V$ & 0 & 1 & 2 & 3 & $\neg$ & 0 \\
\hline 0 & 0 & 0 & 0 & 0 & 0 & 0 & 1 & 2 & 3 & 0 & 3 \\
\hline 1 & 0 & 1 & 0 & 1 & 1 & 1 & 1 & 3 & 3 & 1 & 1 \\
\hline 2 & 0 & 0 & 2 & 2 & 2 & 2 & 3 & 2 & 3 & 2 & 2 \\
\hline 3 & 0 & 1 & 2 & 3 & 3 & 3 & 3 & 3 & 3 & 3 & 0 \\
\hline
\end{tabular}

${ }^{7} I(\Gamma)=\inf \{I(B) \mid B \in \Gamma\}$, so $I(\Gamma) \in D$ iff $I(B) \in D$ for each $B \in \Gamma$. In particular, $\vDash_{\mathrm{M}} A$ ( $A$ is M-valid; $A$ is valid in the matrix M) iff $I(A) \in D$ for all M-interpretations $I$. By $\vDash_{\mathrm{M}}$ we shall refer to the consequence relation defined in $\mathrm{M}$. 
The notions of an MB4-interpretation, MB4-consequence and MB4-validity are defined according to the general Definition 2.5.

The elements in MB4 can be interpreted as noted in Remark 2.7.

Remark 2.7 (On the intuitive meaning of the truth-values in MB4) The truth values $0,1,2$ and 3 can intuitively be interpreted in MB4 as follows. Let $T$ and $F$ represent truth and falsity. Then, $0=F, 1=N$ (either), $2=$ $B$ (oth) and $3=T$. Or, in terms of subsets of $\{T, F\}$, we have: $0=\{F\}, 1=\emptyset$, $2=\{T, F\}$ and $3=\{T\}$.

Next, the notion of a logic determined by a given matrix can be understood as stated in the following definition.

Definition 2.8 (Logics determined by matrices) Let $\mathcal{L}$ be a propositional language, $M$ a matrix for $\mathcal{L}$ and $\vdash_{L}$ a (proof theoretical) consequence relation defined on $\mathcal{L}$. Then, the logic $L$ is determined by $M$ iff for every set of wffs $\Gamma$ and wff $A, \Gamma \vdash_{L} A$ iff $\Gamma \vDash_{M} A$. In particular, the logic $L$ (considered as the set of its theorems) is determined by $M$ iff for every wff $A, \vdash_{L} A$ iff $\vDash_{M} A^{8}$.

\section{Kleene's strong 3-valued matrix with two des- ignated elements}

Kleene's strong 3-valued matrix with two designated elements, MK3, can be regarded as one of the sides of the lattice represented above in Definition 2.6 and it can be defined as shown in Definition 3.1.

Definition 3.1 (MK3) The propositional language is the same as in MB4. Kleene's strong 3-valued matrix with two designated elements, MK3, is the structure $(\mathcal{V}, D, F)$ where $(1) \mathcal{V}=\{0,1,2\}$ and it is ordered as shown in the following lattice

$$
\left.\right|_{0} ^{2}
$$

(2) $D=\{1,2\}$; (3) $\mathrm{F}=\left\{f_{\wedge}, f_{\vee}, f_{\neg}\right\}$ where $f_{\wedge}$ and $f_{\vee}$ are defined similarly as in MB4 and $f_{\neg}$ is an involution with $f_{\neg}(2)=0, f_{\neg}(0)=2$ and $f_{\neg}(1)=1$. We display the tables for $\wedge, \vee$ and $\neg$ :

\footnotetext{
${ }^{8}$ Concerning the logic determined by the matrix MB4, cf. Note 9 .
} 


\begin{tabular}{l|lll}
$\wedge$ & 0 & 1 & 2 \\
\hline 0 & 0 & 0 & 0 \\
1 & 0 & 1 & 1 \\
2 & 0 & 1 & 2
\end{tabular}

\begin{tabular}{l|lll}
$\vee$ & 0 & 1 & 2 \\
\hline 0 & 0 & 1 & 2 \\
1 & 1 & 1 & 2 \\
2 & 2 & 2 & 2
\end{tabular}

\begin{tabular}{l|l}
$\neg$ & 0 \\
\hline 0 & 2 \\
1 & 1 \\
2 & 0
\end{tabular}

The notions of an MK3-interpretation, MK3-consequence and MK3-validity are defined according to the general Definition 2.5.

Notice that we now use 2 for truth and 1 for both, 0 representing falsity as before. On the other hand, conjunction and disjunction are defined similarly as in MB4 and negation is an involution with 1 being its own negation. (We use the symbols 0,1 and 2 -instead of $0,1 / 2$ and 1 because they are convenient for using the tester in [17], in case the reader needs one.)

Now, let K3 be the logic determined by MK3. A BD-semantics for K3 is defined as stated in Definitions 3.2 and 3.3.

Definition 3.2 (K3-models) A K3-model is a structure $(K, I)$ where (i) $K=$ $\{\{T\},\{F\},\{T, F\}\}$; (ii) $I$ is an K3-interpretation from the set of all formulas to $K$, this notion being defined according to the following conditions for each propositional variable $p$ and formulas $A, B:$ (1) $I(p) \in K$; (2a) $T \in I(\neg A)$ iff $F \in I(A)$; (2b) $F \in I(\neg A)$ iff $T \in I(A)$; (3a) $T \in I(A \wedge B)$ iff $T \in I(A)$ and $T \in I(B)$; (3b) $F \in I(A \wedge B)$ iff $F \in I(A)$ or $F \in I(B)$; (4a) $T \in I(A \vee B)$ iff $T \in I(A)$ or $T \in I(B)$; (4b) $F \in I(A \vee B)$ iff $F \in I(A)$ and $F \in I(B)^{9}$.

Definition 3.3 (K3-consequence; K3-validity) For any set of wffs $\Gamma$ and wff $A, \Gamma \vDash_{M} A$ ( $A$ is a consequence of $\Gamma$ in the K3-model $M$ ) iff $T \in I(A)$ if $T \in I(\Gamma)[T \in I(\Gamma)$ iff $\forall A \in \Gamma(T \in I(A)) ; F \in I(\Gamma)$ iff $\exists A \in \Gamma(F \in I(A))]$. In particular, $\vDash_{M} A$ ( $A$ is true in $\left.M\right)$ iff $T \in I(A)$. Then, $\Gamma \vDash_{K 3} A$ ( $A$ is a consequence of $\Gamma$ in K3-semantics) iff $\Gamma \vDash_{M} A$ for each K3-model $M$. In particular, $\vDash_{K 3} A$ ( $A$ is valid in K3-semantics) iff $\vDash_{M} A$ for each K3-model $M$ (i.e., iff $T \in I(A)$ for each K3-model $M)$.

It is well-known that the result of defining the conditional in MK3 similarly as it is defined with disjunction and negation in classical logic is Priest's Logic of Paradox LP (cf. [24], [25], [26]). In this paper, we consider alternative ways of expanding MK3 with a conditional connective. In particular, all natural implicative expansions of MK3 will be investigated. The notion of a natural conditional is defined in the following section ${ }^{10}$.

\footnotetext{
${ }^{9}$ The logic determined by the matrix MB4 can be named B4. It is well-known that B4 is equivalent to Anderson and Belnap's First Degree Entailment Logic FDE (cf. [1], [9], [15]). Then, a BD-semantics for B4, B4-models, can be defined similarly as K3-models are defined, except that the set $K$ is $\{\{T\},\{F\},\{T, F\}, \emptyset\}$ instead of being $K=\{\{T\},\{F\},\{T, F\}\}$ (cf. [9], [10], [14], [15]).

${ }^{10}$ Nevertheless, we think that we have to record the opinion of a referee of the JANCL on the label "Kleene's strong 3-valued matrix with two designated values". According to this referee, MK3 should be named LP since the "fact that Priest's implication is the classical one (and is definable with negation and disjunction) means that the matrix of LP and MK3 are the same".
} 


\section{Natural implicative expansions of MK3}

Following Tomova [34], we define "natural conditionals" as follows.

Definition 4.1 (Natural conditionals) Let $\mathcal{L}$ be a propositional language with $\rightarrow$ among its connectives and $M$ be a matrix for $\mathcal{L}$ where the values $x$ and $y$ represent the supremum and the infimum in $\mathcal{V}$ in the classical sense. Then, an $f \rightarrow$-function on $\mathcal{V}$ defines a natural conditional if the following conditions are satisfied:

1. $f_{\rightarrow}$ coincides with (the $f_{\rightarrow}$-function for) the classical conditional when restricted to the subset $\{x, y\}$ of $\mathcal{V}$.

2. $f \rightarrow$ satisfies Modus Ponens, that is, for any $a, b \in \mathcal{V}$, if $a \rightarrow b \in D$ and $a \in D$, then $b \in D$.

3. For any $a, b \in \mathcal{V}, a \rightarrow b \in D$ if $a \leq b$.

We have:

Proposition 4.2 (Natural conditionals in 3-valued matrices) Let $\mathcal{L}$ be a propositional language and $M$ a 3-valued matrix for $\mathcal{L}$ where $\mathcal{V}$ and $D$ are defined exactly as in MK3. Now, consider the $24 f_{\rightarrow}$-functions defined in the following general table:

$T I$

\begin{tabular}{l|lll}
$\rightarrow$ & 0 & 1 & 2 \\
\hline 0 & 2 & $a_{1}$ & 2 \\
1 & 0 & $a_{2}$ & $a_{3}$ \\
2 & 0 & $b_{1}$ & 2
\end{tabular}

where $a_{i}(1 \leq i \leq 3) \in\{1,2\}$ and $b_{1} \in\{0,1,2\}$. The set of functions contained in TI is the set of all natural conditionals definable in $M$.

Proof. It is obvious.

Next, the notion of a natural implicative 3-valued matrix is defined and a proposition collecting the 24 natural implicative expansions of MK3 is proved.

Definition 4.3 (Natural implicative 3-valued matrices) Let $\mathcal{L}$ be a propositional language with the connective $\rightarrow$. And let $M$ be a 3-valued matrix where $\mathcal{V}$ and $D$ are defined as in Definition 3.1. Moreover, let $f \rightarrow$ be one of the functions (defining one of the conditionals) in TI (in Proposition 4.2). Then, it is said that $M$ is a natural implicative 3-valued matrix ${ }^{11}$.

Proposition 4.4 (Natural implicative expansions of MK3) There are exactly 24 natural implicative expansions of MK3, Mt1, Mt2,..., Mt24, which are defined as follows. Each Mti $(1 \leq i \leq 24)$ is the structure $(\mathcal{V}, D, F)$ where $\mathcal{V}, D, f_{\wedge}, f_{\vee}$ and $f_{\neg}$ are defined exactly as in $M K 3$ (cf. Definition 3.1), whereas

\footnotetext{
${ }^{11}$ Notice that we are supposing that $\mathcal{V}$ is ordered as stated in Definition 3.1 .
} 
$f_{\rightarrow}$ is defined according to the table ti. Tables $t 1$, t2, ..., t24 are displayed below; the notions of an Mti-interpretation, etc. are defined according to the general Definition 2.5.

Proof. Immediate by Proposition 4.2 and Definition 4.3.

List of the 24 tables:

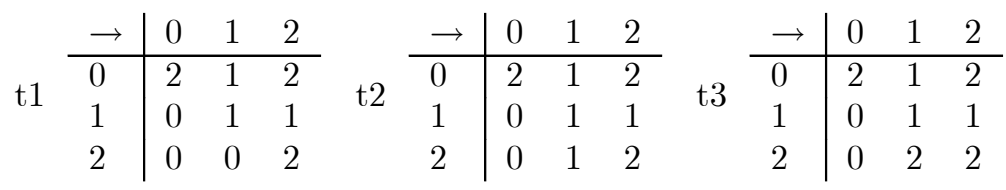

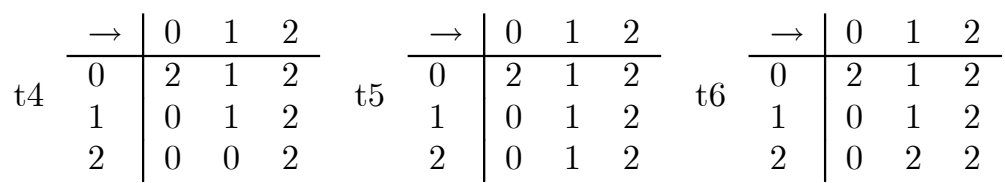

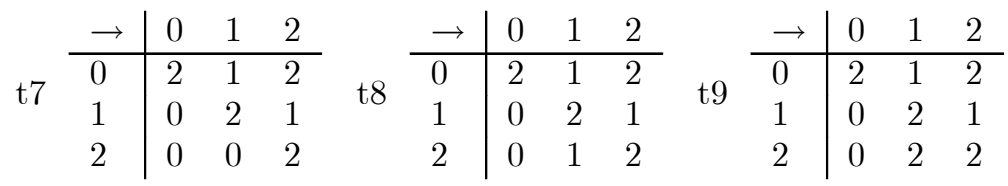

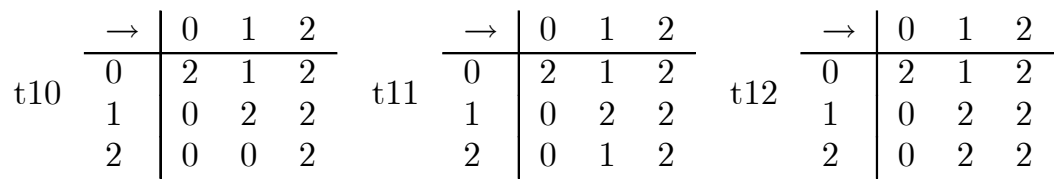

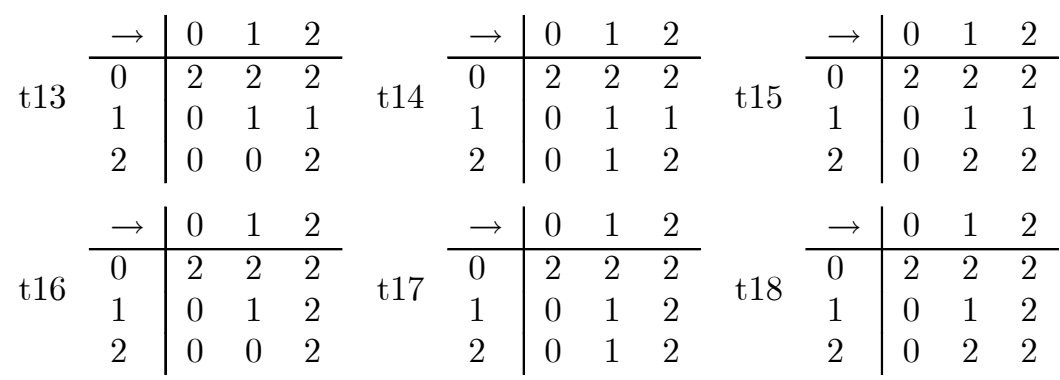

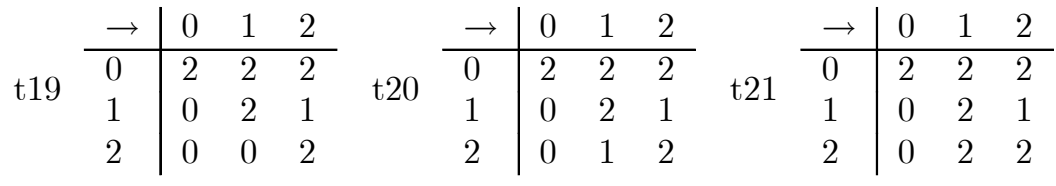

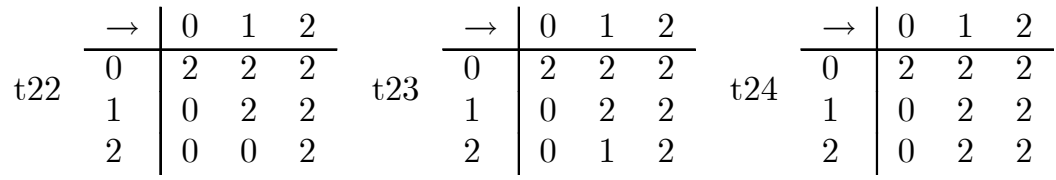


The aim of this paper is to provide an overdetermined BD-semantics for the logic Lt $i$ characterized by each matrix Mt $i(1 \leq i \leq 24)^{12}$.

\section{$5 \quad$ The basic logics $\mathbf{b}^{3}, \mathbf{b}_{1}^{3}, \mathbf{b}_{2}^{3}$}

In this section, the basic logics $\mathrm{b}^{3}, \mathrm{~b}_{1}^{3}, \mathrm{~b}_{2}^{3}$ are defined and some of their properties are proved. The logic $b^{3}$ is axiomatized as follows (the label is intended to abbreviate "basic logic contained in all natural implicative expansions of K3").

Definition 5.1 (The basic logic $\mathbf{b}^{3}$ ) The logic $b^{3}$ is axiomatized with the following axioms and rules of inference:

Axioms:

$$
\begin{aligned}
& \text { A1. }(A \wedge B) \rightarrow A /(A \wedge B) \rightarrow B \\
& \text { A2. }[(A \rightarrow B) \wedge(A \rightarrow C)] \rightarrow[A \rightarrow(B \wedge C)] \\
& \text { A3. } A \rightarrow(A \vee B) / B \rightarrow(A \vee B) \\
& \text { A4. }[(A \rightarrow C) \wedge(B \rightarrow C)] \rightarrow[(A \vee B) \rightarrow C] \\
& \text { A5. }[A \wedge(B \vee C)] \rightarrow[(A \wedge B) \vee(A \wedge C)] \\
& \text { A6. } \neg(A \vee B) \leftrightarrow(\neg A \wedge \neg B) \\
& \text { A7. } \neg(A \wedge B) \leftrightarrow(\neg A \vee \neg B) \\
& \text { A8. } A \leftrightarrow \neg \neg A \\
& \text { A9. } A \vee \neg A \\
& \text { A10. }[(A \rightarrow B) \wedge(B \rightarrow C)] \rightarrow(A \rightarrow C) \\
& \text { A11. }[(A \rightarrow B) \wedge A] \rightarrow B \\
& \text { A12. } A \vee(A \rightarrow B)
\end{aligned}
$$

Rules of inference:

Adjunction: $A \& B \Rightarrow A \wedge B$

Modus Ponens: $A \rightarrow B \& A \Rightarrow B$

Next, the logics $b_{1}^{3}$ and $b_{2}^{3}$ are defined. They are two mutually independent extensions of $\mathrm{b}^{3}$. The former is contained in 16 of the 24 natural implicative expansions of K3; the latter, in the remaining 8 expansions.

Definition 5.2 (The basic logic $\mathbf{b}_{1}^{3}$ ) The logic $b_{1}^{3}$ is axiomatized by adding to $b^{3}$ the following axiom:

$$
\text { A13. } B \rightarrow(A \rightarrow B)
$$

\footnotetext{
${ }^{12}$ A referee of the JANCL proposes to name some of the implications characterized by the tables displayed above. In particular, her(his) proposal is the following: t16, Sobociński's implication; t17, Jáskowski's implication; t18, Carnielli, Marco and de Amo's implication; t22, Rescher's implication; t23, Heyting's implication; t24, Sette's implication (cf. [12], [13], [16], [19] and the references cited in [13] and [19]).
} 
Definition 5.3 (The basic logic $\mathbf{b}_{2}^{3}$ ) The logic $b_{2}^{3}$ is the result of adding the following axioms to $b^{3}$ :

$$
\begin{aligned}
& \text { A14. }[(A \rightarrow B) \wedge \neg B] \rightarrow \neg A \\
& \text { A15. }(\neg A \wedge B) \rightarrow(A \rightarrow B) \\
& \text { A16. } \neg B \vee(A \rightarrow B)
\end{aligned}
$$

Next, we prove some properties of $\mathrm{b}^{3}$ and its extensions. We begin by defining the notion of a $\mathrm{Eb}^{3}$-theory and the classes of $\mathrm{Eb}^{3}$-theories of interest in the present paper. By EL we generally refer to an extension (or an expansion, as the case may be) of the logic L (cf. Definition 2.3).

Definition $5.4\left(\mathbf{E b}^{3}\right.$-theories) Let $L$ be an $E b^{3}$-logic. An L-theory is a set of formulas closed under Adjunction (Adj) and provable L-entailment (L-ent). That is, $\mathcal{T}$ is an L-theory iff we have (1) whenever $A, B \in \mathcal{T}, A \wedge B \in \mathcal{T}$ (Adj); (2) whenever $A \rightarrow B$ is a theorem of $L$ and $A \in \mathcal{T}$, then $B \in \mathcal{T}$ (L-ent).

Definition 5.5 (Classes of $\mathbf{E b}^{3}$-theories) Let $L$ be an $E b^{3}$-logic and $\mathcal{T}$ an $L$-theory. We set (1) $\mathcal{T}$ is prime iff whenever $A \vee B \in \mathcal{T}$, then $A \in \mathcal{T}$ or $B \in \mathcal{T}$; (2) $\mathcal{T}$ is regular iff $\mathcal{T}$ contains all theorems of $L$; (3) $\mathcal{T}$ is trivial iff it contains all wffs; (4) $\mathcal{T}$ is a-consistent (consistent in an absolute sense) iff $\mathcal{T}$ is not trivial; finally, (5) $\mathcal{T}$ is complete iff $A \in \mathcal{T}$ or $\neg A \in \mathcal{T}$ for every wff $A$.

Then, we have:

Proposition 5.6 (Eb ${ }^{3}$-theories are closed under MP) Let $L$ be an $E b^{3}$ logic and $\mathcal{T}$ be an L-theory. Then, $\mathcal{T}$ is closed under Modus Ponens (MP). That is, if $A \rightarrow B \in \mathcal{T}$ and $A \in \mathcal{T}$, then $B \in \mathcal{T}$.

Proof. The proof is immediate by A11 and closure under L-ent and Adj.

Proposition $5.7\left(\mathbf{E b}^{3}\right.$-theories and double negation) Let $L$ be an $E b^{3}$-logic and $\mathcal{T}$ an L-theory. Then, $A \in \mathcal{T}$ iff $\neg \neg A \in \mathcal{T}$.

Proof. Immediate by A8.

Proposition 5.8 (Conjunction and disjunction in prime $\mathbf{E b}^{3}$-theories) Let $L$ be an $E b^{3}$-logic and $\mathcal{T}$ be a prime L-theory. Then, (1) $A \wedge B \in \mathcal{T}$ iff $A \in \mathcal{T}$ and $B \in \mathcal{T}$; (2) $\neg(A \wedge B) \in \mathcal{T} \quad$ iff $\neg A \in \mathcal{T}$ or $\neg B \in \mathcal{T}$; (3) $A \vee B \in \mathcal{T}$ iff $A \in \mathcal{T}$ or $B \in \mathcal{T}$; (4) $\neg(A \vee B) \in \mathcal{T} \quad$ iff $\neg A \in \mathcal{T}$ and $\neg B \in \mathcal{T}$.

Proof. Case 1: by A1 and the fact that $\mathcal{T}$ is closed under Adj; case 2: by A3 and A7; case 3: by $\mathrm{A} 3$ and the fact that $\mathcal{T}$ is prime; case 4 : by $\mathrm{A} 6$ and the fact that $\mathcal{T}$ is closed under Adj.

Proposition 5.9 (Completeness of prime regular $\mathbf{E b}^{3}$-theories) Let $L$ be an $E b^{3}$-logic and $\mathcal{T}$ be a prime regular $L$-theory. Then, $\mathcal{T}$ is complete. 
Proof. Immediate by A9.

Next, we remark some properties of $\mathrm{Eb}_{1}^{3}$-theories and $\mathrm{Eb}_{2}^{3}$-theories.

Proposition $5.10\left(\mathbf{E b}_{2}^{3}\right.$-theories are closed under $\left.\mathbf{M T}\right)$ Let $L$ be an $E b_{2}^{3}$ logic and $\mathcal{T}$ be an L-theory. Then, $\mathcal{T}$ is closed under Modus Tollens (MT). That is, if $A \rightarrow B \in \mathcal{T}$ and $\neg B \in \mathcal{T}$, then $\neg A \in \mathcal{T}$.

Proof. Immediate by A14 and closure of $\mathcal{T}$ under L-ent and Adj.

Proposition 5.11 (The conditional in prime regular $\mathbf{E b}_{1}^{3}$-theories) Let $L$ be an $E b_{1}^{3}$-logic and $\mathcal{T}$ be a prime regular L-theory. Then, $A \rightarrow B \in \mathcal{T}$ iff $A \notin \mathcal{T}$ or $B \in \mathcal{T}$.

Proof. (a) From left to right $(\Rightarrow)$ : Immediate by closure of $\mathrm{Eb}^{3}$-theories under MP. From right to left $(\Leftarrow)$ : Suppose $A \notin \mathcal{T}$. By A12 and regularity of $\mathcal{T}$, $A \vee(A \rightarrow B) \in \mathcal{T}$. Then, $A \rightarrow B \in \mathcal{T}$ by primeness. Suppose now $B \in \mathcal{T}$. Then, $A \rightarrow B \in \mathcal{T}$ is immediate by A13.

Proposition 5.12 (The conditional in prime regular $\mathbf{E b}_{2}^{3}$-theories) Let $L$ be an $E b_{2}^{3}$-logic and $\mathcal{T}$ be a prime regular $L$-theory. Then, $A \rightarrow B \in \mathcal{T}$ iff $A \notin \mathcal{T}$ or $\neg B \notin \mathcal{T}$ or $(\neg A \in \mathcal{T} \quad \& B \in \mathcal{T})$.

Proof. (a) $(\Rightarrow)$ : Suppose (1) $A \rightarrow B \in \mathcal{T}$ and, for reductio, (2) $A \in \mathcal{T}$, $\neg B \in \mathcal{T}$ and $\neg A \notin \mathcal{T}$ or $(3) A \in \mathcal{T}, \neg B \in \mathcal{T}$ and $B \notin \mathcal{T}$. But 2 and 3 are impossible by closure of $\mathcal{T}$ under MP and MT. (b) $(\Leftarrow)$ : If $A \notin \mathcal{T}$ or $\neg B \notin \mathcal{T}$, then $A \rightarrow B \in \mathcal{T}$ follows by regularity and primeness of $\mathcal{T}$ and A12 and A16, respectively. In addition, if $\neg A \in \mathcal{T}$ and $B \in \mathcal{T}$, then $A \rightarrow B \in \mathcal{T}$ follows by A15, given that $\mathcal{T}$ is closed under L-ent and Adj.

This section is ended with the proof of the primeness lemma.

Lemma 5.13 (Primeness) Let $L$ be an $E b^{3}$-logic, $\mathcal{T}$ an L-theory and $A$ a wff such that $A \notin \mathcal{T}$. Then, there is a prime L-theory $\mathcal{T}^{\prime}$ such that $\mathcal{T} \subseteq \mathcal{T}^{\prime}$ and $A \notin \mathcal{T}^{\prime}$

Proof. By using, for example, Kuratowski-Zorn's Lemma, $\mathcal{T}$ is extended to a maximal theory $\mathcal{T}^{\prime}$ such that $A \notin \mathcal{T}^{\prime}$. Then, it is easy to show that $\mathcal{T}^{\prime}$ is prime. (Cf., for example, the proof of Lemma 5.7 in [22]. This proof holds for any logic L that includes B4, provided that L-theories are defined similarly as $\mathrm{Eb}^{3}$-theories, that is, as sets of formulas closed under L-ent and Adj).

\section{Extensions of the basic logics}

In this section, 24 different extensions of $\mathrm{b}^{3}$ are defined. It will be proved that each one of the 24 natural implicative expansions of MK3 defined in Proposition 3.4 characterizes or determines one of these extensions. Sixteen of these extensions of $b^{3}$ contain $b_{1}^{3}$ while $b_{2}^{3}$ is contained in the remaining eight systems. 
The extensions just referred to are axiomatized by using the following list of axioms:

$$
\begin{aligned}
& \text { A17. }(A \wedge \neg A) \rightarrow \neg(A \rightarrow B) \\
& \text { A18. }(B \wedge \neg B) \rightarrow \neg(A \rightarrow B) \\
& \text { A19. }(A \wedge \neg B) \rightarrow \neg(A \rightarrow B) \\
& \text { A20. }[\neg(A \rightarrow B) \wedge \neg A] \rightarrow(A \vee B) \\
& \text { A21. }[\neg(A \rightarrow B) \wedge B] \rightarrow(\neg A \vee \neg B) \\
& \text { A22. } \neg(A \rightarrow B) \rightarrow(A \vee \neg B) \\
& \text { A23. } \neg(A \rightarrow B) \rightarrow(A \vee B) \\
& \text { A24. } \neg(A \rightarrow B) \rightarrow(\neg A \vee \neg B) \\
& \text { A25. }[(B \wedge \neg B) \wedge \neg A] \rightarrow \neg(A \rightarrow B) \\
& \text { A26. } A \rightarrow[B \vee \neg(A \rightarrow B)] \\
& \text { A27. }[\neg(A \rightarrow B) \wedge B] \rightarrow \neg A \\
& \text { A28. }[\neg(A \rightarrow B) \wedge B] \rightarrow \neg B \\
& \text { A29. } \neg(A \rightarrow B) \rightarrow \neg B \\
& \text { A30. } \neg B \rightarrow[\neg A \vee \neg(A \rightarrow B)] \\
& \text { A31. }(A \wedge \neg A) \rightarrow[\neg B \vee \neg(A \rightarrow B)] \\
& \text { A32. }(B \wedge \neg B) \rightarrow[A \vee \neg(A \rightarrow B)] \\
& \text { A33. }[\neg(A \rightarrow B) \wedge[(A \wedge \neg A) \wedge(B \wedge \neg B)]] \rightarrow C \\
& \text { A34. }[\neg(A \rightarrow B) \wedge[(B \wedge \neg B) \wedge A]] \rightarrow C \\
& \text { A35. }[\neg(A \rightarrow B) \wedge[(A \wedge \neg A) \wedge B]] \rightarrow C \\
& \text { A36. }[\neg(A \rightarrow B) \wedge(A \wedge B)] \rightarrow C \\
& \text { A37. }[\neg(A \rightarrow B) \wedge \neg A] \rightarrow A \\
& \text { A38. } \neg(A \rightarrow B) \rightarrow A \\
& \text { A39. } \neg(A \rightarrow B) \rightarrow(A \wedge \neg B) \\
& \text { A40. }[(A \wedge \neg A) \wedge \neg B] \rightarrow \neg(A \rightarrow B) \\
& \text { A41. }[[\neg(A \rightarrow B) \wedge(B \wedge \neg B)] \wedge \neg A] \rightarrow C \\
& \text { A42. }[\neg(A \rightarrow B) \wedge(B \wedge \neg B)] \rightarrow C \\
& \text { A43. }[\neg(A \rightarrow B) \wedge(\neg A \wedge B)] \rightarrow C \\
& \text { A44. }[\neg(A \rightarrow B) \wedge B] \rightarrow C
\end{aligned}
$$

In order to define the $\mathrm{Eb}^{3}$-extensions, we have followed two guidelines: (1) To axiomatize all systems with a common base as wide as possible; (2) to avoid weak rules of inference, since this type of rules complicates the completeness proofs and, especially, the proof of the extension (to prime theories) lemmas.

Definition 6.1 (Extensions of $\mathbf{b}_{1}^{3}$ ) The 16 extensions of $b_{1}^{3}$ are Lt2, Lt3, Lt5, Lt6, Lt8, Lt9, Lt11, Lt12, Lt14, Lt15, Lt17, Lt18, Lt20, Lt21, Lt23 and Lt24. It will be proved that the logic Lti is characterized by the matrix Mti 
$(i \in\{2,3,5,6,8,9,11,12,14,15,17,18,20,21,23,24\})$. These logics are axiomatized by adding the following axioms to $b_{1}^{3}$.

Lt2:

A1\%. $(A \wedge \neg A) \rightarrow \neg(A \rightarrow B)$

A18. $(B \wedge \neg B) \rightarrow \neg(A \rightarrow B)$

A19. $(A \wedge \neg B) \rightarrow \neg(A \rightarrow B)$

A22. $\neg(A \rightarrow B) \rightarrow(A \vee \neg B)$

A23. $\neg(A \rightarrow B) \rightarrow(A \vee B)$

A24. $\neg(A \rightarrow B) \rightarrow(\neg A \vee \neg B)$

Lt3:

A1\%. $(A \wedge \neg A) \rightarrow \neg(A \rightarrow B)$

A22. $\neg(A \rightarrow B) \rightarrow(A \vee \neg B)$

A23. $\neg(A \rightarrow B) \rightarrow(A \vee B)$

A25. $[(B \wedge \neg B) \wedge \neg A] \rightarrow \neg(A \rightarrow$

$B)$

A26. $A \rightarrow[B \vee \neg(A \rightarrow B)]$

A2\%. $[\neg(A \rightarrow B) \wedge B] \rightarrow \neg A$

Lt5:

A18. $(B \wedge \neg B) \rightarrow \neg(A \rightarrow B)$

A19. $(A \wedge \neg B) \rightarrow \neg(A \rightarrow B)$

A23. $\neg(A \rightarrow B) \rightarrow(A \vee B)$

A29. $\neg(A \rightarrow B) \rightarrow \neg B$

Lt6:

A23. $\neg(A \rightarrow B) \rightarrow(A \vee B)$

A25. $[(B \wedge \neg B) \wedge \neg A] \rightarrow \neg(A \rightarrow B)$

A26. $A \rightarrow[B \vee \neg(A \rightarrow B)]$

A27. $[\neg(A \rightarrow B) \wedge B] \rightarrow \neg A$

A29. $\neg(A \rightarrow B) \rightarrow \neg B$
Lt8:

$$
\begin{aligned}
& \text { A22. } \neg(A \rightarrow B) \rightarrow(A \vee \neg B) \\
& \text { A23. } \neg(A \rightarrow B) \rightarrow(A \vee B) \\
& \text { A24. } \neg(A \rightarrow B) \rightarrow(\neg A \vee \neg B) \\
& \text { A26. } A \rightarrow[B \vee \neg(A \rightarrow B)] \\
& \text { A30. } \neg B \rightarrow[\neg A \vee \neg(A \rightarrow B)] \\
& \text { A31. }(A \wedge \neg A) \rightarrow[\neg B \vee \neg(A \rightarrow \\
&B)] \\
& \text { A32. }(B \wedge \neg B) \rightarrow[A \vee \neg(A \rightarrow \\
&B)] \\
& \text { A33. } {[\neg(A \rightarrow B) \wedge[(A \wedge \neg A) \wedge} \\
&(B \wedge \neg B)]] \rightarrow C
\end{aligned}
$$

Lt9:

$$
\begin{aligned}
\text { A22. } & \neg(A \rightarrow B) \rightarrow(A \vee \neg B) \\
\text { A23. } & \neg(A \rightarrow B) \rightarrow(A \vee B) \\
\text { A26. } & A \rightarrow[B \vee \neg(A \rightarrow B)] \\
\text { A2\%. } & {[\neg(A \rightarrow B) \wedge B] \rightarrow \neg A } \\
\text { A31. } & (A \wedge \neg A) \rightarrow[\neg B \vee \neg(A \rightarrow \\
& B)] \\
\text { A32. } & (B \wedge \neg B) \rightarrow[A \vee \neg(A \rightarrow \\
& B)] \\
\text { A34. } & {[\neg(A \rightarrow B) \wedge[(B \wedge \neg B) \wedge} \\
& A]] \rightarrow C
\end{aligned}
$$

Lt11:

$$
\begin{aligned}
& \text { A23. } \neg(A \rightarrow B) \rightarrow(A \vee B) \\
& \text { A26. } A \rightarrow[B \vee \neg(A \rightarrow B)] \\
& \text { A29. } \neg(A \rightarrow B) \rightarrow \neg B \\
& \text { A30. } \neg B \rightarrow[\neg A \vee \neg(A \rightarrow B)] \\
& \text { A32. }(B \wedge \neg B) \rightarrow[A \vee \neg(A \rightarrow \\
&B)] \\
& \text { A35. } {[\neg(A \rightarrow B) \wedge[(A \wedge \neg A) \wedge} \\
&B]] \rightarrow C
\end{aligned}
$$


Lt12:

$$
\begin{aligned}
\text { A23. } \neg(A \rightarrow B) \rightarrow(A \vee B) \\
\text { A26. } A \rightarrow[B \vee \neg(A \rightarrow B)] \\
\text { A29. } \neg(A \rightarrow B) \rightarrow \neg B \\
\text { A32. }(B \wedge \neg B) \rightarrow[A \vee \neg(A \rightarrow \\
\\
\quad B)] \\
\text { A36. }[\neg(A \rightarrow B) \wedge(A \wedge B)] \rightarrow C
\end{aligned}
$$

Lt14:

$$
\begin{aligned}
& \text { A17. }(A \wedge \neg A) \rightarrow \neg(A \rightarrow B) \\
& \text { A19. }(A \wedge \neg B) \rightarrow \neg(A \rightarrow B) \\
& \text { A24. } \neg(A \rightarrow B) \rightarrow(\neg A \vee \neg B) \\
& \text { A37. }[\neg(A \rightarrow B) \wedge \neg A] \rightarrow A
\end{aligned}
$$

\section{Lt15:}

$$
\begin{aligned}
& \text { A17. }(A \wedge \neg A) \rightarrow \neg(A \rightarrow B) \\
& \text { A26. } A \rightarrow[B \vee \neg(A \rightarrow B)] \\
& \text { A27. }[\neg(A \rightarrow B) \wedge B] \rightarrow \neg A \\
& \text { A38. } \neg(A \rightarrow B) \rightarrow A
\end{aligned}
$$

Lt1\%:

$$
\begin{aligned}
& \text { A19. }(A \wedge \neg B) \rightarrow \neg(A \rightarrow B) \\
& \text { A39. } \neg(A \rightarrow B) \rightarrow(A \wedge \neg B)
\end{aligned}
$$

\section{Lt18:}

$$
\begin{aligned}
& \text { A26. } A \rightarrow[B \vee \neg(A \rightarrow B)] \\
& \text { A27. }[\neg(A \rightarrow B) \wedge B] \rightarrow \neg A \\
& \text { A39. } \neg(A \rightarrow B) \rightarrow(A \wedge \neg B) \\
& \text { A40. }[(A \wedge \neg A) \wedge \neg B] \rightarrow \neg(A \rightarrow
\end{aligned}
$$

Lt20:

$$
\begin{aligned}
\text { A24. } \neg(A \rightarrow B) \rightarrow(\neg A \vee \neg B) \\
\text { A26. } A \rightarrow[B \vee \neg(A \rightarrow B)] \\
\text { A30. } \neg B \rightarrow[\neg A \vee \neg(A \rightarrow B)] \\
\text { A31. }[(A \wedge \neg A) \rightarrow[\neg B \vee \neg(A \rightarrow \\
\\
\quad B)] \\
\text { A38. } \neg(A \rightarrow B) \rightarrow A \\
\text { A41. }[\neg(A \rightarrow B) \wedge[(B \wedge \neg B) \wedge \\
\quad \neg A]] \rightarrow C
\end{aligned}
$$

Lt21:

$$
\begin{aligned}
& \text { A26. } A \rightarrow[B \vee \neg(A \rightarrow B)] \\
& \text { A27. } {[\neg(A \rightarrow B) \wedge B] \rightarrow \neg A } \\
& \text { A31. }(A \wedge \neg A) \rightarrow[\neg B \vee \neg(A \rightarrow \\
&B)] \\
& \text { A38. } \neg(A \rightarrow B) \rightarrow A \\
& \text { A42. }[\neg(A \rightarrow B) \wedge(B \wedge \neg B)] \rightarrow \\
& \\
& C
\end{aligned}
$$

\section{Lt23:}

A26. $A \rightarrow[B \vee \neg(A \rightarrow B)]$

A30. $\neg B \rightarrow[\neg A \vee \neg(A \rightarrow B)]$

A39. $\neg(A \rightarrow B) \rightarrow(A \wedge \neg B)$

A43. $[\neg(A \rightarrow B) \wedge(\neg A \wedge B)] \rightarrow$

C

Lt24:

$$
\begin{aligned}
& \text { A26. } A \rightarrow[B \vee \neg(A \rightarrow B)] \\
& \text { A39. } \neg(A \rightarrow B) \rightarrow(A \wedge \neg B) \\
& \text { A44. }[\neg(A \rightarrow B) \wedge B] \rightarrow C
\end{aligned}
$$

Definition 6.2 (Extensions of $\mathbf{b}_{2}^{3}$ ) The 8 extensions of $b_{2}^{3}$ are Lt1, Lt4, Lt7, Lt10, Lt13, Lt16, Lt19 and Lt22. As in the case of the extensions of $b_{1}^{3}$, it will be proved that the logic Lti is characterized by the matrix Mti $(i \in$ $\{1,4,7,10,13,16,19,22\})$. These 8 extensions of $b_{2}^{3}$ are axiomatized by adding the following axioms to $b_{2}^{3}$ : 
Lt1:

A17. $(A \wedge \neg A) \rightarrow \neg(A \rightarrow B)$

A18. $(B \wedge \neg B) \rightarrow \neg(A \rightarrow B)$

A19. $(A \wedge \neg B) \rightarrow \neg(A \rightarrow B)$

A20. $[\neg(A \rightarrow B) \wedge \neg A] \rightarrow(A \vee$

$B)$

A21. $[\neg(A \rightarrow B) \wedge B] \rightarrow(\neg A \vee$ $\neg B)$

A22. $\neg(A \rightarrow B) \rightarrow(A \vee \neg B)$

Lt4:

A18. $(B \wedge \neg B) \rightarrow \neg(A \rightarrow B)$

A19. $(A \wedge \neg B) \rightarrow \neg(A \rightarrow B)$

A20. $[\neg(A \rightarrow B) \wedge \neg A] \rightarrow(A \vee$

$B)$

A22. $\neg(A \rightarrow B) \rightarrow(A \vee \neg B)$

A28. $[\neg(A \rightarrow B) \wedge B] \rightarrow \neg B$

Lt7:

A20. $[\neg(A \rightarrow B) \wedge \neg A] \rightarrow(A \vee$

$B)$

A21. $[\neg(A \rightarrow B) \wedge B] \rightarrow(\neg A \vee$ $\neg B)$

A22. $\neg(A \rightarrow B) \rightarrow(A \vee \neg B)$

A26. $A \rightarrow[B \vee \neg(A \rightarrow B)]$

A30. $\neg B \rightarrow[\neg A \vee \neg(A \rightarrow B)]$

A31. $(A \wedge \neg A) \rightarrow[\neg B \vee \neg(A \rightarrow$ $B)]$

A32. $(B \wedge \neg B) \rightarrow[A \vee \neg(A \rightarrow$ $B)]$

A33. $[\neg(A \rightarrow B) \wedge[(A \wedge \neg A) \wedge$ $(B \wedge \neg B)]] \rightarrow C$

Lt10:

A20. $[\neg(A \rightarrow B) \wedge \neg A] \rightarrow(A \vee$ $B)$

A26. $A \rightarrow[B \vee \neg(A \rightarrow B)]$

A28. $[\neg(A \rightarrow B) \wedge B] \rightarrow \neg B$

A30. $\neg B \rightarrow[\neg A \vee \neg(A \rightarrow B)]$
A32. $(B \wedge \neg B) \rightarrow[A \vee \neg(A \rightarrow$ $B)]$

A35. $[\neg(A \rightarrow B) \wedge[(A \wedge \neg A) \wedge$ $B]] \rightarrow C$

Lt13:

$$
\begin{aligned}
& \text { A17. }(A \wedge \neg A) \rightarrow \neg(A \rightarrow B) \\
& \text { A19. }(A \wedge \neg B) \rightarrow \neg(A \rightarrow B) \\
& \text { A21. }[\neg(A \rightarrow B) \wedge B] \rightarrow(\neg A \vee \\
& \\
& \quad \neg B) \\
& \text { A3\%. }[\neg(A \rightarrow B) \wedge \neg A] \rightarrow A
\end{aligned}
$$

Lt16:

$$
\begin{aligned}
& \text { A19. }(A \wedge \neg B) \rightarrow \neg(A \rightarrow B) \\
& \text { A28. }[\neg(A \rightarrow B) \wedge B] \rightarrow \neg B \\
& \text { A3\%. }[\neg(A \rightarrow B) \wedge \neg A] \rightarrow A
\end{aligned}
$$

Lt19:

A21. $[\neg(A \rightarrow B) \wedge B] \rightarrow(\neg A \vee$ $\neg B)$

A26. $A \rightarrow[B \vee \neg(A \rightarrow B)]$

A30. $\neg B \rightarrow[\neg A \vee \neg(A \rightarrow B)]$

A31. $(A \wedge \neg A) \rightarrow[\neg B \vee \neg(A \rightarrow$ $B)]$

A3\%. $[\neg(A \rightarrow B) \wedge \neg A] \rightarrow A$

A41. $[\neg(A \rightarrow B) \wedge[(B \wedge \neg B) \wedge$ $\neg A]] \rightarrow C$

Lt22:

A26. $A \rightarrow[B \vee \neg(A \rightarrow B)]$

A28. $[\neg(A \rightarrow B) \wedge B] \rightarrow \neg B$

A30. $\neg B \rightarrow[\neg A \vee \neg(A \rightarrow B)]$

A3\%. $[\neg(A \rightarrow B) \wedge \neg A] \rightarrow A$

A43. $[\neg(A \rightarrow B) \wedge(\neg A \wedge B)] \rightarrow$ C 
In what follows, we prove two important propositions on the behavior of negated conditionals in the extensions of $b_{1}^{3}$ and $b_{2}^{3}$ just defined.

Proposition 6.3 (Negated conditionals in $\mathbf{E b}_{1}^{3}$-logics) Let $L$ be an ELtilogic where Lti will refer in each case to one of the extensions of $b_{1}^{3}$ displayed in Definition 6.1. And let $\mathcal{T}$ be a prime, regular and a-consistent $L$-theory. We have:

- ELt2-logics: $\neg(A \rightarrow B) \in \mathcal{T}$ iff $(A \in \mathcal{T} \quad \& \quad \neg A \in \mathcal{T})$ or $(B \in \mathcal{T} \quad \& \quad \neg B \in \mathcal{T})$ or $(A \in \mathcal{T} \quad \& \neg B \in \mathcal{T})$.

- ELt3-logics: $\neg(A \rightarrow B) \in \mathcal{T}$ iff $(A \in \mathcal{T} \quad \& \quad \neg A \in \mathcal{T})$ or $(A \in \mathcal{T} \quad \& \quad B \notin \mathcal{T})$ or $(B \in$ $\mathcal{T} \& \neg B \in \mathcal{T} \& \neg A \in \mathcal{T})$.

- ELt5-logics: $\neg(A \rightarrow B) \in \mathcal{T}$ iff $(A \in \mathcal{T} \quad \& \quad \neg B \in \mathcal{T})$ or $(B \in \mathcal{T} \& \neg B \in \mathcal{T})$.

- ELt6-logics: $\neg(A \rightarrow B) \in \mathcal{T}$ iff $(A \in \mathcal{T} \quad \& \quad B \notin \mathcal{T})$ or $(B \in$ $\mathcal{T} \& \neg B \in \mathcal{T} \& \neg A \in \mathcal{T})$.

- ELt8-logics: $\neg(A \rightarrow B) \in \mathcal{T}$ iff $(A \in \mathcal{T} \quad \& \quad B \notin \mathcal{T})$ or $(\neg A \notin \mathcal{T} \quad \& \quad \neg B \in \mathcal{T})$ or $(A \in$ $\mathcal{T} \quad \& \neg A \in \mathcal{T} \quad \& \neg B \notin \mathcal{T}$ ) or $(B \in \mathcal{T} \quad \& \neg B \in \mathcal{T} \quad \& A \notin \mathcal{T})$.

- ELt9-logics: $\neg(A \rightarrow B) \in \mathcal{T}$ iff $(A \in \mathcal{T} \quad \& \quad B \notin \mathcal{T})$ or $(A \in$ $\mathcal{T} \& \neg A \in \mathcal{T} \quad \& \neg B \notin \mathcal{T}$ ) or $(B \in \mathcal{T} \& \neg B \in \mathcal{T} \& A \notin \mathcal{T})$.

- ELt11-logics: $\neg(A \rightarrow B) \in \mathcal{T}$ iff $(A \in \mathcal{T} \quad \& \quad B \notin \mathcal{T})$ or $(\neg A \notin \mathcal{T} \quad \& \quad \neg B \in \mathcal{T})$ or $(B \in \mathcal{T} \quad \& \neg B \in \mathcal{T} \quad \& \quad A \notin \mathcal{T})$.
- ELt12-logics: $\neg(A \rightarrow B) \in \mathcal{T}$ iff $(A \in \mathcal{T} \quad \& \quad B \notin \mathcal{T})$ or $(B \in \mathcal{T} \quad \& \neg B \in \mathcal{T} \quad \& A \notin \mathcal{T})$.

- ELt14-logics: $\neg(A \rightarrow B) \in \mathcal{T}$ iff $(A \in \mathcal{T} \quad \& \quad \neg A \in \mathcal{T})$ or $(A \in \mathcal{T} \& \neg B \in \mathcal{T})$.

- ELt15-logics: $\neg(A \rightarrow B) \in \mathcal{T}$ iff $(A \in \mathcal{T} \quad \& \quad \neg A \in \mathcal{T})$ or $(A \in \mathcal{T} \quad \& \quad B \notin \mathcal{T})$.

- ELt1\%-logics: $\neg(A \rightarrow B) \in \mathcal{T}$ iff $A \in \mathcal{T} \& \neg B \in \mathcal{T}$.

- ELt18-logics: $\neg(A \rightarrow B) \in \mathcal{T}$ iff $(A \in \mathcal{T} \& B \notin \mathcal{T})$ or $(A \in \mathcal{T} \quad \&$ $\neg A \in \mathcal{T} \quad \& \neg B \in \mathcal{T})$.

- ELt20-logics: $\neg(A \rightarrow B) \in \mathcal{T}$ iff $(A \in \mathcal{T} \quad \& \quad B \notin \mathcal{T})$ or $(\neg A \notin \mathcal{T} \quad \& \quad \neg B \in \mathcal{T})$ or $(A \in$ $\mathcal{T} \& \neg A \in \mathcal{T} \& \neg B \notin \mathcal{T})$.

- ELt21-logics: $\neg(A \rightarrow B) \in \mathcal{T}$ iff $(A \in \mathcal{T} \quad \& \quad B \notin \mathcal{T})$ or $(A \in \mathcal{T} \quad \&$ $\neg A \in \mathcal{T} \quad \& \neg B \notin \mathcal{T})$.

- ELt23-logics: $\neg(A \rightarrow B) \in \mathcal{T}$ iff $(\neg A \notin \mathcal{T} \quad \& \quad \neg B \in \mathcal{T})$ or $(A \in \mathcal{T} \& B \notin \mathcal{T})$.

- ELt24-logics: $\neg(A \rightarrow B) \in \mathcal{T}$ iff $A \in \mathcal{T} \& B \notin \mathcal{T}$.

Proof. It is similar to the proofs of Propositions 5.11 and 5.12. So, it will suffice to prove one case, say case 5 .

Case 5. ELt8-logics:

(a) $(\Rightarrow)$ : Suppose $(1) \neg(A \rightarrow B) \in \mathcal{T}$ and, for reductio, (2) ( $A \notin \mathcal{T}$ or $B \in \mathcal{T}) \&(\neg A \in \mathcal{T}$ or $\neg B \notin \mathcal{T}) \quad \&(A \notin \mathcal{T}$ or $\neg A \notin \mathcal{T}$ or $\neg B \in \mathcal{T}) \quad \& \quad(B \notin \mathcal{T}$ 
or $\neg B \notin \mathcal{T}$ or $A \in \mathcal{T}$ ). There are 36 subcases to consider. The first 18 are the following.

$$
\begin{aligned}
& \text { 1. } A \notin \mathcal{T} \quad \& \neg A \in \mathcal{T} \quad \& \quad A \in \mathcal{T} \quad \& \quad A \notin \mathcal{T} \\
& \text { 2. } A \notin \mathcal{T} \& \neg A \in \mathcal{T} \quad \& \quad A \in \mathcal{T} \quad \& \neg A \notin \mathcal{T} \\
& \text { 3. } A \notin \mathcal{T} \& \neg A \in \mathcal{T} \quad \& \quad A \in \mathcal{T} \quad \& \neg B \in \mathcal{T} \\
& \text { 4. } A \notin \mathcal{T} \& \neg A \in \mathcal{T} \& B \notin \mathcal{T} \& A \notin \mathcal{T} \\
& \text { 5. } A \notin \mathcal{T} \quad \& \neg A \in \mathcal{T} \quad \& \quad B \notin \mathcal{T} \& \neg A \notin \mathcal{T} \\
& \text { 6. } A \notin \mathcal{T} \quad \& \neg A \in \mathcal{T} \quad \& B \notin \mathcal{T} \& \neg B \in \mathcal{T} \\
& \text { 7. } A \notin \mathcal{T} \& \neg A \in \mathcal{T} \quad \& \neg B \notin \mathcal{T} \quad \& \quad A \notin \mathcal{T} \\
& \text { 8. } A \notin \mathcal{T} \& \neg A \in \mathcal{T} \& \neg B \notin \mathcal{T} \& \neg A \notin \mathcal{T} \\
& \text { 9. } A \notin \mathcal{T} \& \neg A \in \mathcal{T} \& \neg B \notin \mathcal{T} \& \neg B \in \mathcal{T} \\
& \text { 10. } A \notin \mathcal{T} \& \neg B \notin \mathcal{T} \& A \in \mathcal{T} \& A \notin \mathcal{T} \\
& \text { 11. } A \notin \mathcal{T} \& \neg B \notin \mathcal{T} \& A \in \mathcal{T} \& \neg A \notin \mathcal{T} \\
& \text { 12. } A \notin \mathcal{T} \& \neg B \notin \mathcal{T} \& A \in \mathcal{T} \quad \& \neg B \in \mathcal{T} \\
& \text { 13. } A \notin \mathcal{T} \& \neg B \notin \mathcal{T} \& B \notin \mathcal{T} \& A \notin \mathcal{T} \\
& \text { 14. } A \notin \mathcal{T} \& \neg B \notin \mathcal{T} \& B \notin \mathcal{T} \& \neg A \notin \mathcal{T} \\
& \text { 15. } A \notin \mathcal{T} \& \neg B \notin \mathcal{T} \& B \notin \mathcal{T} \& \neg B \in \mathcal{T} \\
& \text { 16. } A \notin \mathcal{T} \& \neg B \notin \mathcal{T} \& \neg B \notin \mathcal{T} \& A \notin \mathcal{T} \\
& \text { 17. } A \notin \mathcal{T} \& \neg B \notin \mathcal{T} \& \neg B \notin \mathcal{T} \& \neg A \notin \mathcal{T} \\
& \text { 18. } A \notin \mathcal{T} \quad \& \neg B \notin \mathcal{T} \quad \& \neg B \notin \mathcal{T} \& \neg B \in \mathcal{T}
\end{aligned}
$$

Let us examine these 18 subcases. Now, 1, 2, 3, 5, 8, 9, 10, 11, 12, 15 and 18 are impossible, since each one of them contains a contradiction. Then, 13, 14 and 17 contradict the fact that all prime regular $\mathrm{Eb}^{3}$-theories are complete. So, we are left with subcases $4,6,7$ and 16 , which are proved as follows: subcases 4 and 6 , by A23; and subcases 7 and 16 , by A22. Let us prove, for example, subcase 16. By the hypothesis (1) and A22, we have $A \vee \neg B \in \mathcal{T}$, whence, by primeness, $A \in \mathcal{T}$ or $\neg B \in \mathcal{T}$, contradicting 16 .

Concerning the remaining 18 subcases, they are exactly as 1-18 above, except that $A \notin \mathcal{T}$ (the first member in each conjunction) is replaced for $B \in \mathcal{T}$. Let us name $1^{\prime}-18^{\prime}$ these remaining 18 subcases. Then $1^{\prime}, 2^{\prime}, 4^{\prime}, 5^{\prime}, 6^{\prime}, 8^{\prime}, 9^{\prime}, 10^{\prime}$, $12^{\prime}, 13^{\prime}, 14^{\prime}, 15^{\prime}$ and $18^{\prime}$ contain a contradiction. So, we are left with subcases $3^{\prime}, 7^{\prime}, 11^{\prime}, 16^{\prime}$ and $17^{\prime}$. Subcase $3^{\prime}$ is proved by A33; subcases $7^{\prime}$ and $16^{\prime}$, by A22; finally, subcases $11^{\prime}$ and $17^{\prime}$ by A24. Let us prove, for example, subcase $3^{\prime}$. By the hypothesis (1) and $3^{\prime}$, we have $\neg(A \rightarrow B) \wedge[(A \wedge \neg A) \wedge(B \wedge \neg B)] \in \mathcal{T}$, whence by A31, we get $C \in \mathcal{T}$, for arbitrary $C$, contradicting the a-consistency of $\mathcal{T}$.

(b) $(\Leftarrow)$ Suppose $(1)(A \in \mathcal{T} \quad \& B \notin \mathcal{T})$ or $(2)(\neg A \notin \mathcal{T} \quad \& \neg B \in \mathcal{T})$ or (3) $(A \in \mathcal{T} \quad \& \neg A \in \mathcal{T} \quad \& \neg B \notin \mathcal{T})$ or $(4)(B \in \mathcal{T} \quad \& \neg B \in \mathcal{T} \quad \& \quad A \notin \mathcal{T})$. Then, $\neg(A \rightarrow B) \in \mathcal{T}$ follows by A26, A30, A31 and A32, respectively. Consider, for example, (4). By (4) and A32, we have $A \vee \neg(A \rightarrow B) \in \mathcal{T}$ whence by $A \notin \mathcal{T}$ 
and primeness of $\mathcal{T}, \neg(A \rightarrow B) \in \mathcal{T}$ follows.

Proposition 6.4 (Negated conditionals in $\mathbf{E b}_{2}^{3}$-logics) Let $L$ be an ELtilogic where Lti will refer in each case to one of the extensions of $b_{2}^{3}$ displayed in Definition 6.2. And let $\mathcal{T}$ be a prime, regular and a-consistent L-theory. We have:

- ELt1-logics: $\neg(A \rightarrow B) \in \mathcal{T}$ iff $(A \in \mathcal{T} \quad \& \quad \neg A \in \mathcal{T})$ or $(B \in \mathcal{T} \quad \& \quad \neg B \in \mathcal{T})$ or $(A \in \mathcal{T} \quad \& \neg B \in \mathcal{T})$.

- ELt4-logics: $\neg(A \rightarrow B) \in \mathcal{T}$ iff $(A \in \mathcal{T} \quad \& \quad \neg B \in \mathcal{T})$ or $(B \in \mathcal{T} \quad \& \neg B \in \mathcal{T})$.

- ELt7-logics: $\neg(A \rightarrow B) \in \mathcal{T}$ iff $(A \in \mathcal{T} \quad \& \quad B \notin \mathcal{T})$ or $(\neg A \notin \mathcal{T} \quad \& \quad \neg B \in \mathcal{T})$ or $(A \in$ $\mathcal{T} \& \neg A \in \mathcal{T} \& \neg B \notin \mathcal{T}$ ) or $(B \in \mathcal{T} \quad \& \neg B \in \mathcal{T} \quad \& \quad A \notin \mathcal{T})$.

- ELt10-logics: $\neg(A \rightarrow B) \in \mathcal{T}$ iff $(A \in \mathcal{T} \quad \& \quad B \notin \mathcal{T})$ or $(\neg A \notin \mathcal{T} \quad \& \quad \neg B \in \mathcal{T})$ or $(B \in \mathcal{T} \quad \& \neg B \in \mathcal{T} \quad \& \quad A \notin \mathcal{T})$.

- ELt13-logics: $\neg(A \rightarrow B) \in \mathcal{T}$ iff $(A \in \mathcal{T} \quad \& \quad \neg A \in \mathcal{T})$ or $(A \in \mathcal{T} \quad \& \neg B \in \mathcal{T})$.

- ELt16-logics: $\neg(A \rightarrow B) \in T$ iff $A \in T \& \neg B \in T$.

- ELt19-logics: $\neg(A \rightarrow B) \in \mathcal{T}$ iff $(A \in \mathcal{T} \quad \& \quad B \notin \mathcal{T})$ or $(\neg A \notin \mathcal{T} \quad \& \quad \neg B \in \mathcal{T})$ or $(A \in$ $\mathcal{T} \& \neg A \in \mathcal{T} \& \neg B \notin \mathcal{T})$.

- ELt22-logics: $\neg(A \rightarrow B) \in \mathcal{T}$ iff $(A \in \mathcal{T} \quad \& \quad B \notin \mathcal{T})$ or $(\neg A \notin \mathcal{T} \quad \& \quad \neg B \in \mathcal{T})$.

Proof. It is similar to that of Proposition 6.3 and it is left to the reader.

\section{Belnap-Dunn semantics for the Lt $i$-logics}

We will define two types of models: $\mathrm{Eb}_{1}^{3}$-models for extensions of $\mathrm{b}_{1}^{3}$, and $\mathrm{Eb}_{2}^{3}{ }^{-}$ models for extensions of $b_{2}^{3}$. Clauses for conjunction, disjunction and negation are the same as in K3-models (cf. Definition 3.2). According to Definitions 7.1 and 7.2, two types of models are generally distinguished by different necessary and suficient conditions for assigning truth, $T$ (see the (5a) clauses), and falsity, $F$ (see the (5b) clauses), to conditionals. The general notions of ELti-consequence and ELti-validity are defined similarly as in K3-models (cf. Definition 3.3).

Definition 7.1 ( $\mathbf{E b}_{1}^{3}$-models) An Eb $b_{1}^{3}$-model is a structure $(K, I)$ where (i) $K=\{\{T\},\{F\},\{T, F\}\}$ and (ii) $I$ is an $E b_{1}^{3}$-interpretation from the set of all formulas to $K$, this notion being defined similarly as in K3-models (cf. Definition 3.2), save for the addition of clauses (5a) and (5b) concerning the conditional. Clause (5a) $T \in I(A \rightarrow B)$ iff $T \notin I(A)$ or $T \in I(B)$. Clause (5b) for each one of the $E b_{1}^{3}$-models is as follows: 
- Lt2-models: (5b) $F \in I(A \rightarrow B)$ iff $[T \in I(A) \quad \& \quad F \in I(A)]$ or $[T \in I(B) \quad \& \quad F \in I(B)]$ or $[T \in I(A) \& F \in I(B)]$.

- Lt3-models: (5b) $F \in I(A \rightarrow B)$ iff $[T \in I(A) \quad \& \quad T \notin I(B)]$ or $[T \in I(A) \quad \& \quad F \in I(A)]$ or $[T \in I(B) \quad \& \quad F \in I(B) \quad \&$ $F \in I(A)]$

- Lt5-models: (5b) $F \in I(A \rightarrow B)$ iff $[T \in I(B) \quad \& \quad F \in I(B)]$ or $[T \in I(A) \& F \in I(B)]$.

- Lt6-models: (5b) $F \in I(A \rightarrow B)$ iff $[T \in I(A) \quad \& \quad T \notin I(B)]$ or $[T \in I(B) \& F \in I(B) \& F \in$ $I(A)]$.

- Lt8-models: (5b) $F \in I(A \rightarrow B)$ iff $[T \in I(A) \quad \& \quad T \notin I(B)]$ or $[F \notin I(A) \quad \& \quad F \in I(B)]$ or $[T \in I(A) \quad \& \quad F \in I(A) \quad \&$ $F \notin I(B)]$ or $[T \in I(B) \& F \in$ $I(B) \& T \notin I(A)]$.

- Lt9-models: (5b) $F \in I(A \rightarrow B)$ iff $[T \in I(A) \quad \& \quad T \notin I(B)]$ or $[T \in I(A) \quad \& \quad F \in I(A) \quad \&$ $F \notin I(B)]$ or $[T \in I(B) \& F \in$ $I(B) \& T \notin I(A)]$.

- Lt11-models: (5b) $F \in I(A \rightarrow$ B) iff $[T \in I(A) \quad \& \quad T \notin I(B)]$ or $[F \notin I(A) \quad \& \quad F \in I(B)]$ or $[T \in I(B) \& F \in I(B) \& T \notin$ $I(A)]$.
- Lt12-models: (5b) $F \in I(A \rightarrow$ $B)$ iff $[T \in I(A) \quad \& \quad T \notin I(B)]$ or $[T \in I(B) \quad \& \quad F \in I(B) \quad \&$ $T \notin I(A)]$.

- Lt14-models: (5b) $F \in I(A \rightarrow$ B) iff $[T \in I(A) \quad \& \quad F \in I(A)]$ or $[T \in I(A) \& F \in I(B)]$.

- Lt15-models: (5b) $F \in I(A \rightarrow$ B) iff $[T \in I(A) \quad \& \quad F \in I(A)]$ or $[T \in I(A) \& T \notin I(B)]$.

- Lt17-models: (5b) $F \in I(A \rightarrow$ $B)$ iff $T \in I(A) \& F \in I(B)$.

- Lt18-models: (5b) $F \in I(A \rightarrow$ $B)$ iff $[T \in I(A) \quad \& \quad T \notin I(B)]$ or $[T \in I(A) \quad \& \quad F \in I(A) \quad \&$ $F \in I(B)]$.

- Lt20-models: (5b) $F \in I(A \rightarrow$ $B)$ iff $[T \in I(A) \quad \& \quad T \notin I(B)]$ or $[F \notin I(A) \quad \& \quad F \in I(B)]$ or $[T \in I(A) \& F \in I(A) \& F \notin$ $I(B)]$

- Lt21-models: (5b) $F \in I(A \rightarrow$ B) iff $[T \in I(A) \quad \& \quad T \notin I(B)]$ or $[T \in I(A) \quad \& \quad F \in I(A) \quad \&$ $F \notin I(B)]$.

- Lt23-models: (5b) $F \in I(A \rightarrow$ $B)$ iff $[T \in I(A) \quad \& \quad T \notin I(B)]$ or $[F \notin I(A) \& F \in I(B)]$.

- Lt24-models: (5b) $F \in I(A \rightarrow$ B) iff $[T \in I(A) \& T \notin I(B)]$.

Definition 7.2 ( $\mathbf{E b}_{2}^{3}$-models) An Eb $b_{2}^{3}$-model is a structure $(K, I)$ where $K$ and $I$ are defined similarly as in $E b_{1}^{3}$-models, save for clauses (5a) and (5b), which are now as follows. Clause (5a) $T \in I(A \rightarrow B)$ iff $T \notin I(A)$ or $F \notin I(B)$ or $(F \in I(A) \& T \in I(B))$. Clause (5b) for each one of the Eb $b_{2}^{3}$-models is as follows: 
- Lt1-models: (5b) $F \in I(A \rightarrow B)$ iff $[T \in I(A) \quad \& \quad F \in I(A)]$ or $[T \in I(B) \quad \& \quad F \in I(B)]$ or $[T \in I(A) \& F \in I(B)]$.

- Lt4models: (5b) $F \in I(A \rightarrow B)$ iff $[T \in I(B) \quad \& \quad F \in I(B)]$ or $[T \in I(A) \& F \in I(B)]$.

- Lt7-models: (5b) $F \in I(A \rightarrow B)$ iff $[T \in I(A) \quad \& \quad T \notin I(B)]$ or $[F \notin I(A) \quad \& \quad F \in I(B)]$ or $[T \in I(A) \quad \& \quad F \in I(A) \quad \&$ $F \notin I(B)]$ or $[T \in I(B) \& F \in$ $I(B) \& T \notin I(A)]$.

- Lt10-models: (5b) $F \in I(A \rightarrow$ B) iff $[T \in I(A) \quad \& \quad T \notin I(B)]$ or $[F \notin I(A) \quad \& \quad F \in I(B)]$ or
$[T \in I(B) \& F \in I(B) \& T \notin$ $I(A)]$.

- Lt13-models: (5b) $F \in I(A \rightarrow$ $B)$ iff $[T \in I(A) \quad \& \quad F \in I(A)]$ or $[T \in I(A) \& F \in I(B)]$.

- Lt16-models: (5b) $F \in I(A \rightarrow$ B) iff $T \in I(A) \& F \in I(B)$.

- Lt19-models: (5b) $F \in I(A \rightarrow$ $B)$ iff $[T \in I(A) \quad \& \quad T \notin I(B)]$ or $[F \notin I(A) \quad \& \quad F \in I(B)]$ or $[T \in I(A) \& F \in I(A) \& F \notin$ $I(B)]$

- Lt22-models: (5b) $F \in I(A \rightarrow$ $B)$ iff $[T \in I(A) \quad \& \quad T \notin I(B)]$ or $[F \notin I(A) \& F \in I(B)]$.

Definition 7.3 (Lt $i$-consequence, Lt $i$-validity) Let $M$ be an Lti-model $(1 \leq$ $i \leq 24)$. For any set of wffs $\Gamma$ and wff $A, \Gamma \vDash_{M} A$ ( $A$ is a consequence of $\Gamma$ in the Lti-model $M)$ iff $T \in I(A)$ whenever $T \in I(\Gamma)[T \in I(\Gamma)$ iff $\forall A \in \Gamma(T \in I(A)) ; F \in I(\Gamma)$ iff $\exists A \in \Gamma(F \in I(A))]$. Then, $\Gamma \vDash_{L t i} A$ (A is a consequence of $\Gamma$ in Lti-semantics) iff $\Gamma \vDash_{M} A$ for each Lti-model M. In particular, $\vDash_{L t i} A$ (A is valid in Lti-semantics) iff $\vDash_{M}$ A for each Lti-model $M$ (i.e., iff $T \in I(A)$ for each Lti-model $M)$. (By $\vDash_{L t i}$ we shall refer to the relation just defined.)

Next, it is proved that the consequence relation $\vDash_{\mathrm{Mti}}$ (cf. Proposition 4.4 and Definition 3.3) and the relation $\vDash_{\mathrm{Lt} i}$ just defined are coextensive. Then, soundness of each Lt $i$-logic w.r.t. both consequence relations will immediately follow.

Proposition 7.4 (Coextensiveness of $\vDash_{\mathrm{Mt} i}$ and $\left.\vDash_{\mathbf{L t} i}\right)$ For any $i(1 \leq i \leq$ 24), set of wffs $\Gamma$ and wff $A, \Gamma \vDash_{M t i} A$ iff $\Gamma \vDash_{L t i} A$. In particular, $\vDash_{M t i} A$ iff $\vDash_{L t i} A$.

Proof. For any $i(1 \leq i \leq 24)$, let $I$ be an Mti-interpretation. Then, define the Lt $i$-interpretation $I^{\prime}$ corresponding to $I$ as follows: for each propositional variable $p$ set

1. $I^{\prime}(p)=\{T\}$ iff $I(p)=2$.

2. $I^{\prime}(p)=\{T, F\}$ iff $I(p)=1$.

3. $I^{\prime}(p)=\{F\}$ iff $I(p)=0$. 
Next, $I^{\prime}$ interprets complex formulas according to clauses (2a), (2b), (3a), (3b), (4a), (4b) (Definitions 7.1 and 7.2) and (5a) (Definition 7.1 for Eb $\mathrm{b}_{1}^{3}$-models; Definition 7.2 for $\mathrm{Eb}_{2}^{3}$-models). In addition, negated conditionals are interpreted according to clause (5b) (Definition 7.1 for $\mathrm{Eb}_{1}^{3}$-models; Definition 7.2 for $\mathrm{Eb}_{2}^{3}{ }^{-}$ models). Then, by an easy induction we immediately have, for any wff $A$ : (a) $T \in I^{\prime}(A)$ iff $I(A)=2$ or $I(A)=1$; and (b) $F \in I^{\prime}(A)$ iff $I(A)=0$ or $I(A)=1$. In general, for any set of wffs $\Gamma$, we have: (a) $T \in I^{\prime}(\Gamma)$ iff $I(\Gamma)=2$ or $I(\Gamma)=1$; and (b) $F \in I^{\prime}(\Gamma)$ iff $I(\Gamma)=0$ or $I(\Gamma)=1$.

On the other hand, given an Lti-interpretation $I^{\prime}$, the Mti-interpretation $I$ corresponding to $I^{\prime}$ can be defined in a similar way and with similar results.

Once these facts stated, the proof of Proposition 7.4 follows by Propositions 4.4 and Definitions 3.3 and 7.3.

Now, we can prove soundness.

Theorem 7.5 (Soundness of Lt $i$ w.r.t. $\vDash_{\mathbf{M t} i}$ and $\left.\vDash_{\mathbf{L t} i}\right)$ For any $i(1 \leq i \leq$ 24), set of formulas $\Gamma$ and formula $A$, if $\Gamma \vdash_{L t i} A$, then (1) $\Gamma \vDash_{M t i} A$ and (2) $\Gamma \vDash_{L t i} A$.

Proof. (1) Given a particular logic Lti, it is easy to check that the rules Adj and MP preserve Mt $i$-validity, whereas the axioms of Lt $i$ are assigned either 2 or else 1 by any Mti-interpretation $I$. Consequently, if $\Gamma \vdash_{\mathrm{Lt} i} A$, then $\Gamma \vDash_{\mathrm{Mt} i}$ A. (2) Then (2) is immediate by (1) and Proposition 7.4. Finally, if $\Gamma$ is the empty set, the proof is similar ${ }^{13}$.

\section{Completeness of the Lt $i$-logics}

We shall prove the completeness of Lt $i(1 \leq i \leq 24)$ (cf. Definitions 6.1 and $6.2)$ w.r.t. both $\vDash_{\mathrm{Mt} i}$ and $\vDash_{\mathrm{Lt} i}$.

Completeness w.r.t. $\vDash_{\mathrm{Lt} i}$ is proved by means of a canonical model construction. Then, completeness w.r.t. $\vDash_{\mathrm{Mt} i}$ follows immediately by Proposition 7.4.

We begin by the definition of canonical $\mathrm{Eb}^{3}$-models. Then, canonical Ltimodels are a particular case of canonical $\mathrm{Eb}^{3}$-models. It will be proved that if $A$ is not derivable from $\Gamma$ in Lt $i$, then $A$ does not follow from $\Gamma$ in some canonical Lt $i$-model. The concept of a canonical Lt $i$-model is based upon the notion of a $\mathcal{T}$-interpretation. In what follows, we refer by Lt $i(1 \leq i \leq 24)$ to any of the 24 extensions of $\mathrm{b}^{3}$ defined in section 6 .

Definition 8.1 ( $\mathcal{T}$-interpretations) Let $L$ be an $E b^{3}$-logic and $K$ be the set $\{\{T\},\{F\},\{T, F\}\}$ as in Definition 3.2. And let $\mathcal{T}$ be a prime, regular and a-consistent L-theory. Then, the function I from the set of all formulas to $K$ is defined as follows: for each propositional variable $p$, we set (a) $T \in I(p)$ iff $p \in \mathcal{T}$; (b) $F \in I(p)$ iff $\neg p \in \mathcal{T}$. Next, I assigns a member of $K$ to each formula $A$ according to the corresponding conditions 2, 3, 4 and 5 in Definitions 7.1 and 7.2. Then, it is said that $I$ is a $\mathcal{T}$-interpretation.

\footnotetext{
${ }^{13}$ In case a tester is needed, the reader con use that in [17].
} 
Definition 8.2 (Canonical $\mathbf{E b}^{3}$-models) Let $L$ be an $E b^{3}$-logic. A canonical $L$-model is a structure $\left(K, I_{\mathcal{T}}\right)$ where $K$ is defined as in Definition 3.2 and $I_{\mathcal{T}}$ is a $\mathcal{T}$-interpretation built upon a prime, regular and a-consistent $L$-theory $\mathcal{T}$.

Definition 8.3 (The canonical relation $\vDash_{I_{\mathcal{T}}}$ ) Let $L$ be an $E b^{3}$-logic and ( $K$, $\left.I_{\mathcal{T}}\right)$ a canonical L-model. The canonical relation $\vDash_{I_{\mathcal{T}}}$ is defined as follows. For any set of wffs $\Gamma$ and wff $A, \Gamma \vDash_{I_{\mathcal{T}}} A$ ( $A$ is a consequence of $\Gamma$ in the canonical $L$-model $\left.\left(K, I_{\mathcal{T}}\right)\right)$ iff $T \in I_{\mathcal{T}}(A)$ whenever $T \in I_{\mathcal{T}}(\Gamma)$. In particular, $\vDash_{I_{\mathcal{T}}} A(A$ is true in the canonical L-model $\left.\left(K, I_{\mathcal{T}}\right)\right)$ iff $T \in I_{\mathcal{T}}(A)$.

By Definitions 8.2 and 8.3, it is clear that any canonical L-model is an Lmodel.

Proposition 8.4 (Any canonical L-model is an L-model) Let $L$ be an $E b^{3}$ logic and $M=\left(K, I_{\mathcal{T}}\right)$ a canonical L-model. Then, $M$ is indeed an L-model.

Proof. It follows immediately by Definitions 8.2 and 8.3. Notice that each propositional variable — and so, each wff - can be assigned $\{T\},\{F\}$ or $\{T, F\}$ since $\mathcal{T}$, although complete (cf. Proposition 5.9), is not required to be consistent in the classical sense.

Given Proposition 8.4, Lemma 8.5 is the essential fact we have to prove in order to prove completeness. In this lemma, conditions (a) and (b) in Definition 8.1 are generalized to all wffs. The proof of Lemma 8.5 leans on Propositions 5.11, 5.12, 6.3 and 6.4, in their turn based upon Propositions 5.6 through 5.10 and Lemma 5.13 .

Lemma 8.5 ( $\mathcal{T}$-interpreting the set of wffs) Let $L$ be an $E b^{3}$-logic and $I$ be a $\mathcal{T}$-interpretation defined on the $L$-theory $\mathcal{T}$. For each wff $A$, we have: (1) $T \in I(A)$ iff $A \in \mathcal{T}$; (2) $F \in I(A)$ iff $\neg A \in \mathcal{T}$.

Proof. Induction on the length of $A$ (H.I. abbreviates "hypothesis of induction").

(I) $A$ is a propositional variable, a conjunction, a disjunction or a negative formula (clauses cited in points (b), (c) and (d) below refer to clauses in Definition 7.1 or Definition 7.2). (a) $A$ is a propositional variable: by conditions (a) and (b) in Definition 8.1. (b) $A$ is of the form $\neg B$ : (i) $T \in I(\neg B)$ iff (clause 2a) $F \in I(B)$ iff (H.I.) $\neg B \in \mathcal{T}$. (ii) $F \in I(\neg B)$ iff (clause 2b) $T \in I(B)$ iff (H.I.) $B \in \mathcal{T}$ iff (Proposition 5.7) $\neg \neg B \in \mathcal{T}$. (c) $A$ is of the form $B \wedge C$ : (i) $T \in I(B \wedge C)$ iff (clause 3a) $T \in I(B)$ and $T \in I(C)$ iff (H.I.) $B \in \mathcal{T}$ and $C \in \mathcal{T}$ iff (Proposition 5.8) $B \wedge C \in \mathcal{T}$. (ii) $F \in I(B \wedge C$ ) iff (clause 3b) $F \in I(B)$ or $F \in I(C)$ iff (H.I.) $\neg B \in \mathcal{T}$ or $\neg C \in \mathcal{T}$ iff (Proposition 5.8) $\neg(B \wedge C) \in \mathcal{T}$. (d) $A$ is of the form $B \vee C$ : the proof is similar to (c) by using clauses 4a, $4 \mathrm{~b}$ and Proposition 5.8.

(II) $A$ is a conditional $B \rightarrow C$ assigned $T$ by I.

(IIi) Lt $i$ is an $\mathrm{Eb}_{1}^{3}$-logic: $T \in I(B \rightarrow C)$ iff (clause 5 a in Definition 7.1) $T \notin I(B)$ or $T \in I(C)$ iff (H.I.) $B \notin \mathcal{T}$ or $C \in \mathcal{T}$ iff (Proposition 5.11) $B \rightarrow C \in \mathcal{T}$. 
(IIii) Lt $i$ is an $\mathrm{Eb}_{2}^{3}$-logic: $T \in I(B \rightarrow C)$ iff (clause 5 a in Definition 7.2) $[T \notin I(B)$ or $F \notin I(C)]$ or $[F \in I(B)$ and $T \in I(C)]$ iff (H.I.) $[B \notin \mathcal{T}$ or $\neg C \notin \mathcal{T}]$ or $[\neg B \in \mathcal{T}$ and $C \in \mathcal{T}]$ iff (Proposition 5.12) $B \rightarrow C \in \mathcal{T}$.

(III) $A$ is a conditional $B \rightarrow C$ assigned $F$ by $I$. We have to consider 24 different cases, but we think that a couple of examples will be sufficient.

(IIIi) Lt $i$ is an $\mathrm{Eb}_{1}^{3}$-logic: Let Lt $i$ be, say, Lt12. We have $F \in I(B \rightarrow C)$ iff (clause 5b in Definition 7.1) $[T \in I(B)$ and $T \notin I(C)]$ or $[T \in I(C)$ and $F \in I(C)$ and $T \notin I(B)]$ iff (H.I.) $[B \in \mathcal{T}$ and $C \notin \mathcal{T}]$ or $[C \in \mathcal{T}$ and $\neg C \in \mathcal{T}$ and $B \notin \mathcal{T}$ ] iff (Proposition 6.3) $\neg(B \rightarrow C) \in \mathcal{T}$.

(IIIii) Lt $i$ is an $\mathrm{Eb}_{2}^{3}$-logic: Let Lt $i$ be, for instance, Lt22. We have $F \in I(B \rightarrow$ $C$ ) iff (clause 5b in Definition 7.2) $[T \in I(B)$ and $T \notin I(C)]$ or $[F \notin I(B)$ and $F \in I(C)]$ iff (H.I.) $[B \in \mathcal{T}$ and $C \notin \mathcal{T}]$ or $[\neg B \notin \mathcal{T}$ and $\neg C \in \mathcal{T}]$ iff (Proposition 6.4) $\neg(B \rightarrow C) \in \mathcal{T}$.

Next, we recall the notion of set of consequences of a given set of formulas $\Gamma$ in Lt $i$ and then we prove completeness.

Definition 8.6 (The set of consequences of $\Gamma$ in Lt $i$ ) The set of consequences in Lti of a set of wffs $\Gamma$ (in symbols $C n \Gamma[L t i]$ ) is defined as follows: $C n \Gamma[L t i]=$ $\left\{A \mid \Gamma \vdash_{L t i} A\right\}$.

We note the following remark.

Remark 8.7 (The set of consequences of $\Gamma$ in Lt $i$ is a regular theory) It is obvious that for any $\Gamma, C n \Gamma[L t i]$ is closed under Adj and MP and contains all theorems of Lti. Consequently, it is closed under Lti-entailment.

Theorem 8.8 (Completeness of Lt $i$-logics) For any $i(1 \leq i \leq 24)$, set of formulas $\Gamma$ and formula $A$, (1) if $\Gamma \vDash_{L t i} A$, then $\Gamma \vdash_{L t i} A$; (2) if $\Gamma \vDash_{M t i} A$, then $\Gamma \vdash_{L t i} A$.

Proof. (1) Suppose there are set of wffs $\Gamma$ and wff $A$ such that $\Gamma \nvdash_{\mathrm{Lt} i} A$. We prove $\Gamma \nvdash_{\mathrm{Lt} i} A$. If $\Gamma \nvdash_{\mathrm{Lt} i} A$, clearly $A \notin \mathrm{Cn} \Gamma[\mathrm{Lt} i]$. Then, by the Primeness Lemma (Lemma 5.13), there is a prime Lti-theory $\mathcal{T}$ such that $\Gamma \subseteq \mathcal{T}[\Gamma \subseteq$ $\mathrm{Cn} \Gamma[\mathrm{Lt} i] \subseteq \mathcal{T}]$ and $A \notin \mathcal{T}$. Thus $\mathcal{T}$ is regular (by Remark 8.7) and a-consistent (since $A \notin \mathcal{T})$. Then, $\mathcal{T}$ generates a $\mathcal{T}$-interpretation $I_{\mathcal{T}}$ such that $T \in I_{\mathcal{T}}(\Gamma)$ but $T \notin I_{\mathcal{T}}(A)$ (cf. Lemma 8.5). Consequently, $\Gamma \nvdash_{I_{\mathcal{T}}} A$ (cf. Definition 8.3 and Proposition 8.4) and, finally, $\Gamma \nvdash_{\mathrm{Lt} i} A$, by Definition 7.3, QED. (2) Completeness w.r.t. $\vDash_{\mathrm{Mt} i}$ is immediate by (1) and Proposition 7.4.

If $\Gamma$ is the empty set, let Lt $i$ be the set of all theorems of Lti. Then Lt $i \nvdash_{\mathrm{Lt} i} A$ and we can proceed similarly as in cases (1) and (2) above.

\section{Concluding remarks}

The paper is ended with some remarks. 
1. Most of the Lt $i$-logics defined above had not been brought to light before (to the best of our knowledge). However, there is a couple of famous 3valued logics among them. Lt16 is the logic RM3, the 3-valued extension of the quasi-relevant logic RM (cf. [11]); and Lt17 is the logic Pac (which abbreviates "paraconsistency", firstly defined by Asenjo in 1954 (cf. [4], $[5],[24],[25])$.

2. Given the axiomatizations provided in Definitions 6.1 and 6.2 , it is possible to define all Lt $i$-logics more conspicuously and economically than in Definitions 6.1 and 6.2. For instance, consider Example 9.1 where Lt22 and Lt23 are defined as negation expansions of the positive fragment of Lewis' S4 (cf. [18]) and positive intuitionistic logic, respectively.

\section{Example 9.1 Lt22}

Axioms: (a1) $A \rightarrow A$; (a2) $(A \wedge B) \rightarrow A /(A \wedge B) \rightarrow B$; (a3) $[(A \rightarrow$ $B) \wedge(A \rightarrow C)] \rightarrow[A \rightarrow(B \wedge C)] ;(a 4) A \rightarrow(A \vee B) / B \rightarrow(A \vee B)$; $(a 5)[(A \rightarrow C) \wedge(B \rightarrow C)] \rightarrow[(A \vee B) \rightarrow C] ;(a 6)[A \wedge(B \vee C)] \rightarrow$ $[(A \wedge B) \vee(A \wedge C)] ;($ a $)[A \rightarrow(B \rightarrow C)] \rightarrow[(A \rightarrow B) \rightarrow(A \rightarrow C)]$; $(a 8)(A \rightarrow B) \rightarrow[C \rightarrow(A \rightarrow B)]$; (a9) $(A \rightarrow \neg B) \rightarrow(B \rightarrow \neg A)$; (a10) $(\neg A \rightarrow B) \rightarrow(\neg B \rightarrow A) ;($ a11) $A \vee \neg A ;($ a12) $(\neg A \wedge B) \rightarrow(A \rightarrow B)$; (a13) $\neg A \rightarrow[A \vee(A \rightarrow B)] ;($ a14 $)[\neg(A \rightarrow B) \wedge(\neg A \wedge B)] \rightarrow C$.

Rules of inference: (Modus Ponens) $A \rightarrow B \& A \Rightarrow B$; (Adjunction) $A \& B \Rightarrow A \wedge B$.

Lt23

Axioms: a1-a7 of Lt22 plus (a8) $A \rightarrow(B \rightarrow A)$; (a9) $A \rightarrow \neg \neg A$; (a10) $\neg \neg A \rightarrow A$; (a11) $A \vee \neg A$; (a12) $A \rightarrow[B \vee \neg(A \rightarrow B)] ;($ a13) $\neg B \rightarrow$ $[\neg A \vee \neg(A \rightarrow B)] ;($ a14 $) \neg(A \rightarrow B) \rightarrow(A \wedge \neg B) ;($ a15 $)[\neg(A \rightarrow B) \wedge$ $(\neg A \wedge B)] \rightarrow C$.

Rules: $M P$ and Adj.

3. It is possible to endow non-natural implicative logics with a BD-semantics. For instance, in Example 9.2 we have a negation expansion of classical positive propositional logic not included in classical propositional logic.

Example 9.2 Consider, for example, the expansion of MK3 based upon the following table for the conditional (1 and 2 are designated values)

\begin{tabular}{l|lll}
$\rightarrow$ & 0 & 1 & 2 \\
\hline 0 & 1 & 1 & 1 \\
1 & 0 & 1 & 1 \\
2 & 0 & 2 & 1
\end{tabular}

The logic determined by this expansion can be axiomatized by adding to $b_{1}^{3}$ the following axioms: $A \vee \neg A ;[\neg(A \rightarrow B) \wedge(B \wedge \neg B)] \rightarrow \neg A ;(B \vee \neg A) \vee$ $\neg(A \rightarrow B) ;(\neg A \vee \neg B) \vee \neg(A \rightarrow B)$ and $\neg A \rightarrow \neg(A \rightarrow B)$, but the last two axioms are not, of course, classical tautologies. 
4. All $\mathrm{Eb}_{1}^{3}$-logics contain classical positive propositional logic but lack the rule Contraposition (if $A \rightarrow B$, then $\neg B \rightarrow \neg A$ ); in their turn, all $\mathrm{Eb}_{2}^{3}-$ logics, except Lt16 and Lt22, lack the rule Prefixing (if $B \rightarrow C$, then $(A \rightarrow B) \rightarrow(B \rightarrow C)$ ) or the rule Suffixing (if $A \rightarrow B$, then $(B \rightarrow C) \rightarrow$ $(A \rightarrow C)$ ) (Lt1, Lt4, Lt7 and Lt10 lack Prefixing and Lt13 and Lt19 lack Suffixing). Consequently, Sylvan and Plumwood's minimal logic $\mathrm{B}_{\mathrm{M}}$ (cf. [32]) is not contained in any of the Lti-logics, except Lt16 and Lt22.

5. All Lti-logics are paraconsistent in the sense that the rule ECQ (if $A \wedge \neg A$, then $B$ ) does not hold in any of them (cf. [26], [27]).

6. A referee of the JANCL called out our attention to some work related to the research recorded in our paper. In particular, Kooi and Tamminga [21] study truth-functional extensions of LP (cf. [24] and [25]) in a natural deduction setting, whereas Petrukhin and Shangin [23] investigate some implicational extensions of LP, including extensions by natural implications in the sense of Tomova [34]. Also, Thomas [33] has to be mentioned. In this paper, an extension of LP with Rescher's implication is proposed. Future work on the topic of the present paper could consist in the study of the relations the papers mentioned maintain to each other.

Acknowledgements. - This work is supported by the Spanish Ministry of Economy, Industry and Competitiveness under Grants [FFI2014-53919-P; FFI201782878-P]. - We sincerely thank two referees of the JANCL for their comments and suggestions on a previous version of this paper.

\section{References}

[1] Anderson, A. R., Belnap, N. D. J. (1975). Entailment. The Logic of Relevance and Necessity, vol I. Princeton, NJ: Princeton University Press.

[2] Arieli, O., Avron, A. (1996). Reasoning with logical bilattices. Journal of Logic, Language and Information, 5(1), 25-63.

[3] Arieli, O., Avron, A. (1998). The value of the four values. Artificial Intelligence, 102, 97-141.

[4] Asenjo, F. G. (1954). La Idea de un Cálculo de Antinomias. Seminario Matemático. Universidad Nacional de la Plata.

[5] Asenjo, F. G. (1966). A calculus of antinomies. Notre Dame Journal of Formal Logic, 7(1), 103-105. http://doi.org/10.1305/ndjfl/1093958482.

[6] Avron, A. (1986). On an implicational connective of RM. Notre Dame Journal of Formal Logic, 27(2), 201-209.

[7] Avron, A. (1991). Natural 3-Valued Logics-Characterization and Proof Theory. Journal of Symbolic Logic, 56(1), 276-294. 
[8] Belnap, N. D. Jr. (1960). Entailment and relevance. The Journal of Symbolic Logic, 25(2), 144-146.

[9] Belnap, N. D. Jr. (1977a). A useful four-valued logic. In G. Epstein, J. M. Dunn (Eds.), Modern Uses of Multiple-Valued Logic (pp. 8-37). D. Reidel Publishing Co., Dordrecht.

[10] Belnap, N. D. Jr. (1977b). How a computer should think. In G. Ryle (Ed.), Contemporary Aspects of Philosophy (pp. 30-55). Oriel Press Ltd., Stocksfield.

[11] Brady, R. T. (1982). Completeness Proofs for the Systems RM3 and BN4. Logique et Analyse, 25, 9-32.

[12] Carnielli, W., Marcos, J., Amo, S. de. (2000). Formal inconsistency and evolutionary databases. Logic and Logical Philosophy, 8, 115-152. https://doi.org/10.12775/LLP.2000.008

[13] Ciucci, D., Dubois, D. (2014). Three-Valued Logics, Uncertainty Management and Rough Sets. In J. F. Peters, A. Skowron (Eds.), Transactions on Rough Sets XVII. Lecture Notes in Computer Science, vol 8375 (pp. 1-32). Springer, Berlin, Heidelberg.

[14] Dunn, J. M. (1976). Intuitive semantics for first-degree entailments and "coupled trees." Philosophical Studies, 29, 149-168.

[15] Dunn, J. M. (2000). Partiality and its Dual. Studia Logica, 66(1), 5-40. http://doi.org/10.1023/A:1026740726955.

[16] Gaines, B. R. (1976). Foundations of fuzzy reasoning. International Journal of Man-Machine Studies, 8(6), 623-668. https://doi.org/10.1016/S00207373(76)80027-2

[17] González, C. (2012). MaTest. Retrieved from http://ceguel.es/matest (last accessed 04/01/2018).

[18] Hacking, I. (1963). What is Strict Implication? Journal of Symbolic Logic, 28(1), 51-71.

[19] Karpenko, A. S. (2004). Jaśkowski's criterion and three-valued paraconsistent logics. Logic and Logical Philosophy, 7, 81-86. https://doi.org/10.12775/LLP.1999.006

[20] Kleene, S. C. (1962). Introduction to Metamathematics. North Holland. Reprinted Ishi Press, 2009.

[21] Kooi, B., Tamminga, A. (2012). Completeness via correspondece for extensions of the logic of paradox. The Review of Symbolic Logic, 5(4), 720-730. 
[22] Méndez, J. M., Robles, G. (2016). The logic determined by Smiley's matrix for Anderson and Belnap's First Degree Entailment Logic. Journal of Applied Non-Classical Logics, 26(1), 47-68. http://doi.org/10.1080/11663081.2016.1153930.

[23] Petrukhin, Y., Shangin, V. (2017). Automated correspondence analysis for the binary extensions of the logic of paradox. The Review of Symbolic Logic, 10(4), 756-781. https://doi.org/10.1017/S1755020317000156.

[24] Priest, G. (1979). Logic of Paradox. Journal of Philosophical Logic, 8 (1), 219-241.

[25] Priest, G. (1984). Logic of paradox revisited. Journal of Philosophical Logic, 13(2), 153-179. https://doi.org/10.1007/BF00453020.

[26] Priest, G. (2002). Paraconsistent Logic. In D. M. Gabbay, F. Guenthner (Eds.), Handbook of Philosophical Logic vol. 6 (pp. 287-393). Springer, Dordrecht.

[27] Priest, G., Tanaka, K. and Weber, Z. (2017). Paraconsistent Logic. The Stanford Encyclopedia of Philosophy (Fall 2017 Edition), E. N. Zalta (Ed.), $\mathrm{URL}=<$ https://plato.stanford.edu/archives/fall2017/

entries/logic-paraconsistent/ $>$.

[28] Robles, G. (2014). A simple Henkin-style completeness proof for Gödel 3-valued logic G3. Logic and Logical Philosophy, 23(4), 371-390. http://doi.org/10.12775/LLP.2014.001.

[29] Robles, G., Méndez, J. M. (2014). A paraconsistent 3-valued logic related to Gödel logic G3. Logic Journal of the IGPL, 22(4), 515-538. http://doi.org/10.1093/jigpal/jzt046.

[30] Robles, G., Méndez, J. M. (In preparation). Belnap-Dunn semantics for natural implicative expansions of Kleene's strong three-valued matrix II. Only one designated value.

[31] Robles, G., Salto, F., Méndez, J. M. (2014). Dual Equivalent Two-valued Under-determined and Over-determined Interpretations for Lukasiewicz's 3-valued Logic Ł3. Journal of Philosophical Logic, 43(2/3), 303-332. http://doi.org/10.1007/s10992-012-9264-0.

[32] Sylvan, R., Plumwood, V. (2003). Non-normal relevant logics. In R. Brady (Ed.), Relevant logics and their rivals, vol. II (pp. 10-16). Ashgate, Aldershot and Burlington: Western Philosophy Series.

[33] Thomas, N. (2018), $L P \Rightarrow$ : Extending $L P$ with a strong conditional operator. Cornell University Library. Unpublished manuscript.

[34] Tomova, N. (2012). A Lattice of implicative extensions of regular Kleene's logics. Reports on Mathematical Logic, 47, 173-182. http://doi.org/10.4467/20842589RM.12.008.0689. 